\title{
Research Paper \\ The Effectiveness of Emotion-Focused Therapy on Emotion Regulation Styles and Severity of Obsessive-Compulsive Symptoms in Women With Obsessive-Compul- sive Disorder
}

\section{${ }^{*}$ Leila Shameli ${ }^{1}$, Mahnaz Mehrabizadeh Honarmand ${ }^{2} \odot$, Abdozahra Naa'mi ${ }^{3} \odot$, Iran Davodi ${ }^{4}$}

1. PhD. in Psychology, Assistant Professor, Department of Psychology, Faculty of Literature and Humanities, Salman Farsi University of Kazeroon, Kazeroun, Iran. 2. PhD. in Psychology, Professor, Department of Psychology, Faculty of Education and Psychology, Shahid Chamran University of Ahvaz, Ahwaz, Iran.

3. PhD. in Psychology, Associate Professor, Department of Psychology, Faculty of Education and Psychology, Shahid Chamran University of Ahvaz, Ahwaz, Iran

4. PhD. in Psychology, Assistant Professor, Department of Psychology, Faculty of Education and Psychology, Shahid Chamran University of Ahvaz, Ahwaz, Iran.

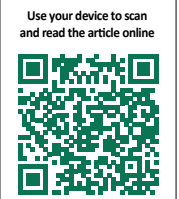

citation Shameli L, Mehrabizadeh Honarmand M, Naa'mi A, Davodi I. [The Effectiveness of Emotion-Focused Therapy on Emotion Regulation Styles and Severity of Obsessive-Compulsive Symptoms in Women With Obsessive-Compulsive Disorder (Persian)]. Iranian Journal of Psychiatry and Clinical Psychology. 2019; 24(4):356-369. http://dx.doi.org/10.32598/ijpcp.24.4.456

http://dx.doi.org/10.32598/ijpcp.24.4.456

Received: 27 Mar 2018

Accepted: $17 \mathrm{Jul} 2018$

Available Online: $01 \operatorname{Jan} 2019$

Keywords:

Emotion-focused

therapy, Emotion regulation styles, Obsessive-Compulsive Disorder (OCD), Severity of obsessive-compulsive symptoms

\section{A B STRACT}

Objectives This study aimed to investigate the effect of emotion-focused therapy on emotional regulation styles and severity of obsessive-compulsive symptoms in women with Obsessive-Compulsive Disorder (OCD). Methods The present study has a quasi-experimental design with pre-test-post-test and control group with follow-up. The statistical population included all women with OCD referring to clinics and counseling centers of Ahvaz City, Iran. In order to select the eligible samples, 30 women were recruited from the patients referred to the treatment centers by convenience sampling method, but some of them were excluded due to discontinuation of treatment sessions. Finally, a sample of 24 patients were evaluated in experimental group (12 subjects) and control group (12 subjects). To collect data, we used the Yale-Brown obsessive-compulsive scale and affective styles questionnaire. The obtained data were analyzed using covariance analysis in SPSS V. 22. Results Multivariate ANOVA test results showed that there was significant difference between test and study groups in terms of concealing style ( $P \leq 0.01, F=7.70)$ and severity of obsessive-compulsive symptoms ( $P \leq 0.001$, $F=20.48$ ) after intervention. Moreover, between female patients in both group, a significant difference was found with respect to concealing style $(P \leq 0.05, F=2.60)$ and severity of obsessive-compulsive symptoms ( $\mathrm{P} \leq 0.001, \mathrm{~F}=29.99$ ) during follow-up period.

Conclusion Based on the results, emotion-focused therapy is an effective treatment to reduce the symptoms of obsessive-compulsive symptoms in patients with OCD.

\section{Extended Abstract}

\section{Introduction}

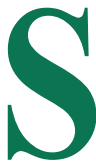

ymptoms of Obsessive-Compulsive Disorder (OCD) are not pleasing obsessive thoughts and compulsive actions. The results of the studies on patients with anxiety and mood disorders indicate that in comparison with the control group, these individuals present a range of emotional problems such as severe reactions to emotional experience and disturbance in the correction of negative emotions. Emotional responses should be regulated, because emotion setting has different consequences in one's life, especially in social and compromising issues.

During research on various patients, including those with OCD, defects in emotional regulation styles have been reported as one of their major problems. Apply-

* Corresponding Author:

Leila Shameli, PhD.

Address: Department of Psychology, Faculty of Literature and Humanities, Salman Farsi University of Kazeroon, Kazeroun, Iran.

Tel: +98 (933) 6598441

E-mail: Ishameli29@yahoo.com 
ing a therapeutic approach that helps patients with their abnormal emotional experiences will be beneficial. This kind of therapy that directly deals with emotions and tries to overcome emotional problems is an EmotionFocused Therapy (EFT). It helps patients to enrich their emotional knowledge, work (not confront with excitement), change and improve them. Research indicates lack of such approaches for patients with OCD. The present research seeks to answer the question of whether EFT affects the emotional regulation styles and the severity of obsessive-compulsive symptoms in patients with OCD at the post-test and follow-up stage.

\section{Method}

The statistical population of this study was all women with OCD referring to clinics and counseling centers of Ahwaz City, Iran. To select the eligible samples, the suitable candidates were recruited by referring to the psychiatric centers and clinics in Ahvaz and enrolling the patients with the diagnosis of OCD and other criteria (at least third grade middle school, aged between 18 and 35 years, no drug intake since one month before beginning treatment). For definitive diagnosis, the researcher conducted a clinical interview with them and implemented the Yale-Brown scale. After meeting the criteria and obtaining at least 16 score of Yale-Brown scale, 30 women were selected by available sampling method and randomly assigned into two groups (experimental and control groups).

Six individuals in the sample group were excluded from the eight 90-minute treatment sessions (that were performed twice a week) because they did not completely participate in sessions. Finally, a sample of 24 women with OCD in experimental group (12 subjects) and control group (12 subjects) were evaluated with Affective Styles Questionnaire (ASS), Yale-Brown Obsessive-Compulsive Scale (YBOCS) and EFT protocol in three stages (pre-test, post-test and follow-up) and then the results were compared using covariance test.

\section{Results}

The results indicate a significant difference between the women in the experimental group and control group in terms of concealing style from emotional regulation styles in the post-test and follow-up stages. Also, there was a difference between two groups in terms of severity of obsessive-compulsive symptoms. Considering the mean score of concealing style in the experimental group compared to control group and also the mean score of severity of obsessive-compulsive symptoms in the experimental group compared to the control group, EFT increased concealing style and reduced the severity of obsessive-compulsive symptoms in women suffering from OCD in the experimental group.

\section{Discussion}

The purpose of this study was to investigate the effectiveness of EFT on emotion regulation styles and severity of obsessive-compulsive symptoms in women with OCD. The results of multivariate covariance analysis indicate that EFT among the three emotion regulation styles has a significant effect only on the concealing style in post-test and follow-up stages. The effectiveness of the treatment was to enhance the style of concealing and reduced severity of obsessive-compulsive symptoms in the experimental group compared to the control group.

The process of EFT protocol is to lead the patient toward internal control (instead of external control). Hence, we did not expect that the concealing style of emotion regulation exaggerated because of treatment. In addition, it can be pointed out that patients were required at the end of the therapeutic process to use practical solutions, rather than to use obsessive-compulsive strategies. Consequently, over time, obsessive-compulsive patients consider the use of concealing style as a practical way of controlling their emotions, in order to increase their level of awareness and responsibility by reflecting on their own inner ties.

To explain the reduction in the severity of obsessive-compulsive symptoms, it can be noted that EFT has principles that help patients overcome their feelings of guilt and maladaptive shame. Researchers have identified these feelings as important factors in the continuation of OCD. So that if obsessive patients can reduce their high levels of self-criticism and feelings of shame during the course of treatment, the likelihood of the continuation of symptoms of this disorder diminishes.

In sum, EFT helps patients become aware of their emotions, and rebuild abnormal cognitive-emotional schemas, which are often the basis of anxiety symptoms. These findings potentially provide important implications for practitioners and therapists to treat obsessive-compulsive patients. One of the implications of this study is to provide a clear prospect for clinicians who in their treatment sessions with obsessive-compulsive patients do not pay attention to the primary and secondary excitement of patients, and do not inform them of abnormal emotions that occur during obsessions and compulsion behavior. Other benefit of this treatment is its low cost due to the short-term and groupbased nature of the protocol, as Ellis believes that the best 
treatment is to teach patients how to deal with life's problems in a limited time.

\section{Ethical Considerations}

\section{Compliance with ethical guidelines}

In this research, the form of ethical considerations was filled by the patients and was followed by the researchers.

\section{Funding}

This article is from the $\mathrm{PhD}$. dissertation of Leila Shameli in Department of Psychology, Shahid Chamran University of Ahvaz.

\section{Authors contributions}

The authors contributions is as follows: Conceptualization: all authors; Methodology: Leila Shameli; Validation: all authors; Analysis: all authors; Research: Leila Shameli; Sources: Leila Shameli; Writing draft: Leila Shameli; Editing and finalizing: Leila Shameli; and Project management: all authors.

\section{Conflict of interest}

The authors declare no conflict of interest

\section{Acknowledgements}

In the end, we appreciate the director of Taravat Counseling Center, who provided the consultation center space. 


\title{
اثربخشى درمان متمركز بر هيجان، بر سبكهاى تنظيم هيجان و شدت علائم وسواس در زنان مبتلابه اختلال وسواسى داجبارى متركي
}

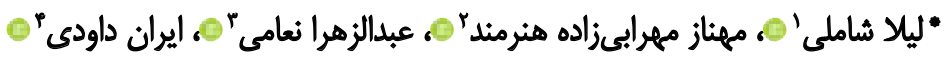

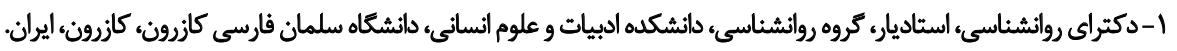

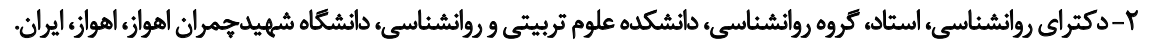

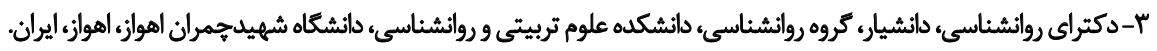

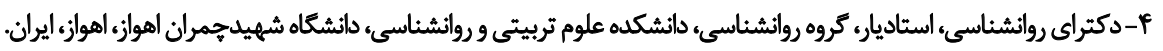

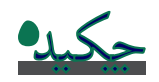

هداف هدف اين يُووهش بررسى اثربخشى درمان مثمركز بر هيجان، بر سبكهاى تنظيم هيجان و شدت علاثم وسواس در زنان مبتلابه اختلال وسواسى اجبارى است.

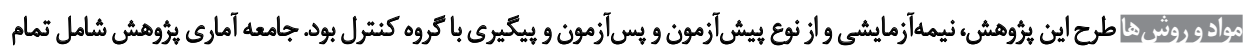

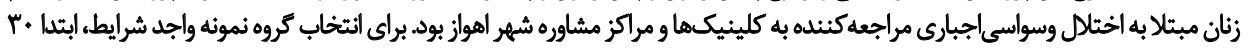

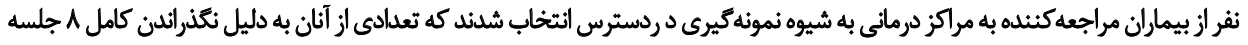

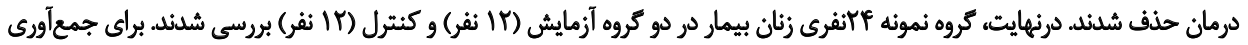

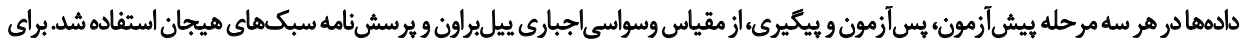

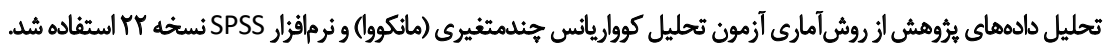

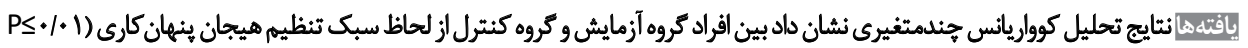

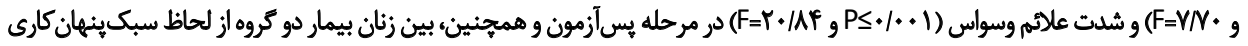

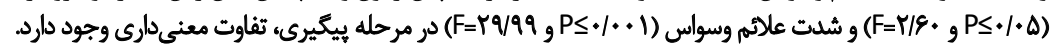

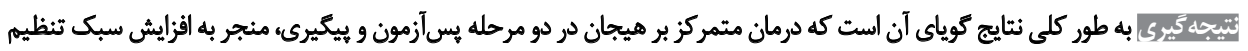

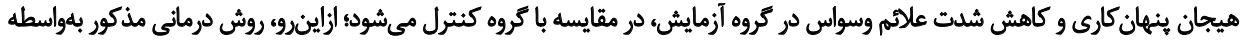

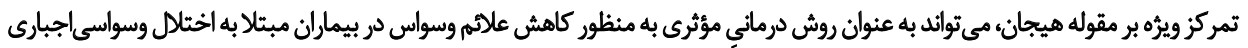
مورد توجه قرار كيرد.
\end{abstract}

تاريخ دريافت: V Vروردين IFaV

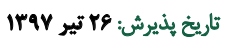

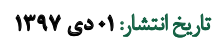

: اojogh

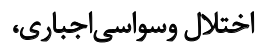
درمان متمركز بر هيجان، سبك هاى تنظيهم هيجان،

شدت علائم وسواس تيفي هيج

نشخوار افكار و تكرار اعمال ادامه مي دهد تا شايد از ناراحتى اش

dalo

كاسته شود. هدف اعمال وسواسى، كاهش يريشانى مرتبط با افكار

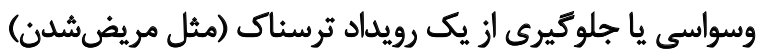

در نسخه ينجم راهنماى تشخيصى و آمارى اختلالهاى

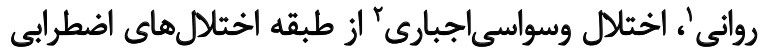

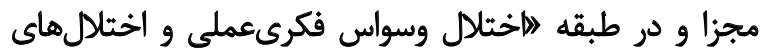

با وجود اين، يا اين اعمال در مسير منطقى مربوط به حوادث

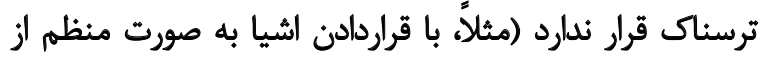
آسيبرسيدن به فرد عزيزى جلوكيرى مي شود) يا آشكارا افراطى مئى

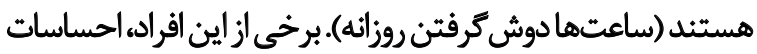

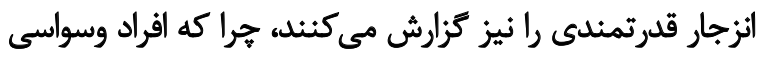

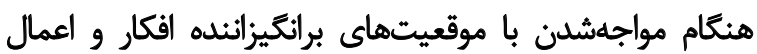

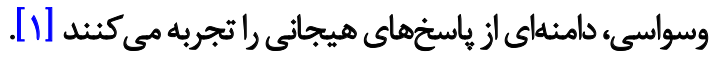

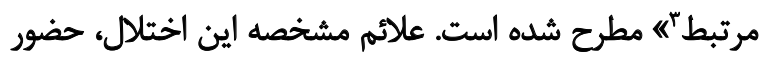
افكار وسواسى و اعمال اجبارى است كه ويرّكى عمده آنها آنها (افكار

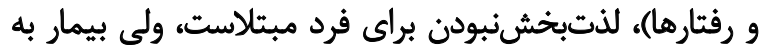

1. Diagnostic and Statistical Manual of Mental Disorders $\left(5^{\text {th }} \mathrm{ed}\right)$ (DSM-5)

2. Obsessive-Compulsive Disorder (OCD)

3. Obsessive-Compulsive Disorder and Related Disorders (OCRD) 
كمك مي شود به واسطه افزودن به دانش هيجانى إنان و كاركردن

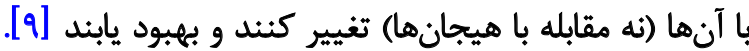

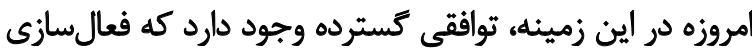

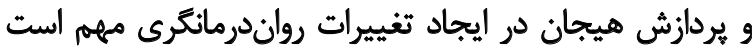

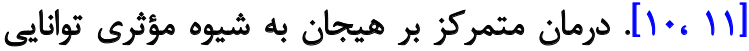

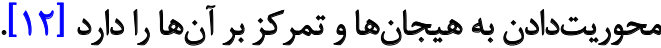
اين روش درمانى كه اساس ساختار روانى و تعيينكنينده

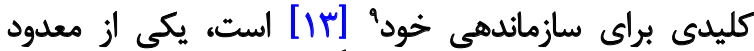

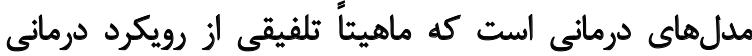

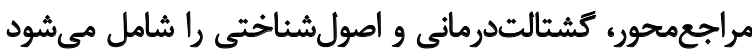

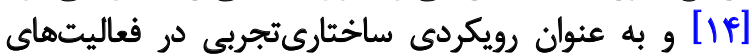

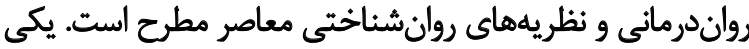

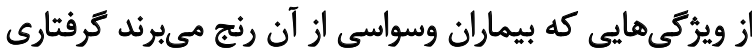

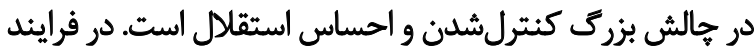

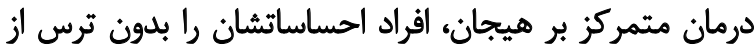

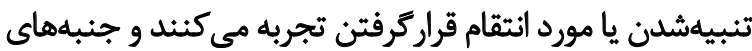

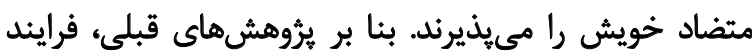

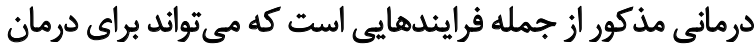

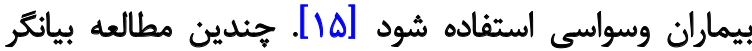

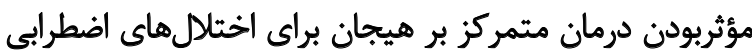

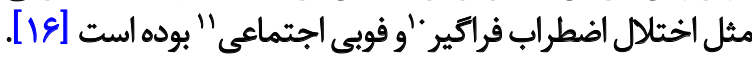

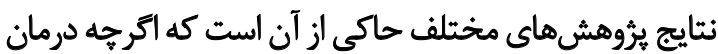

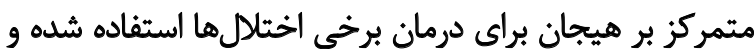

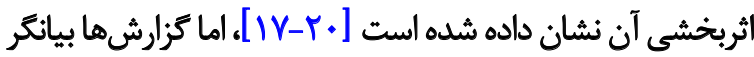

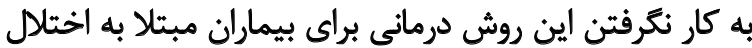

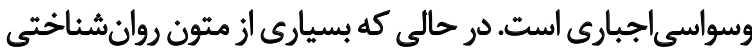

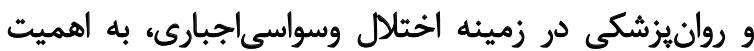

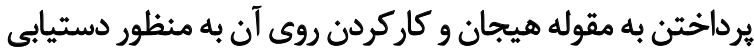

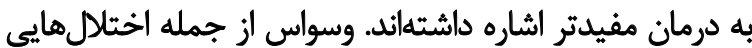

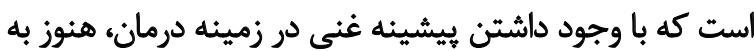

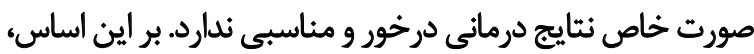

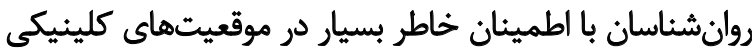

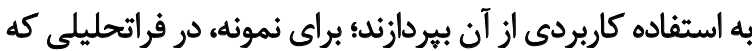

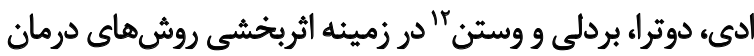

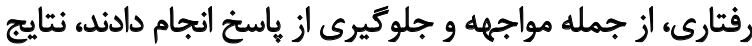

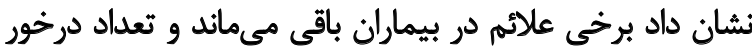

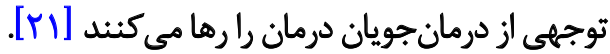
آنجه جبران اين كافئبودن درمان راخواهد كرد، به كاركيرى

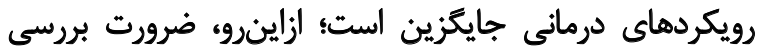

9. Self

10. General anxiety disorder

11. Gocial phobia

12. Eddy, Dutra, Bradley \& Westen

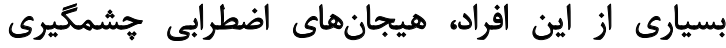

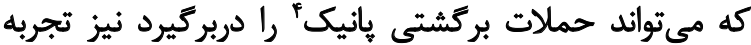

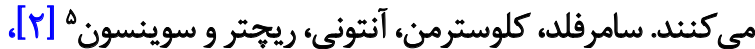

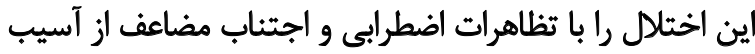

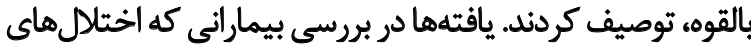

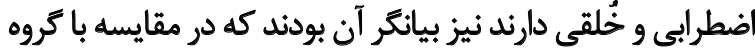

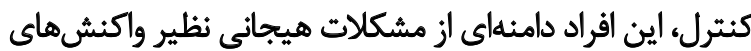

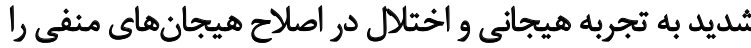

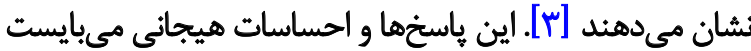

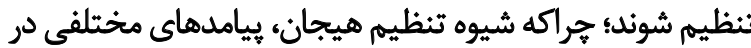

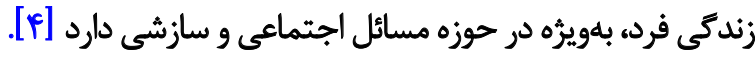
به باور هافمن و كاشدان " ثوانايى تنظيم و ساز كارى هيجانها

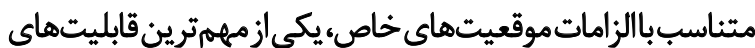

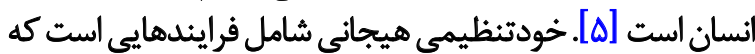

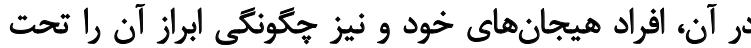

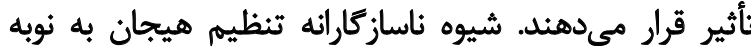

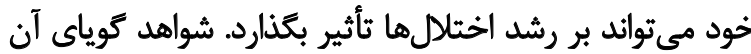

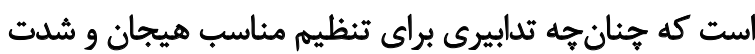

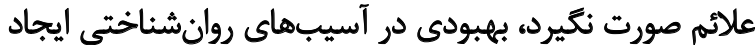

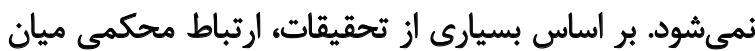

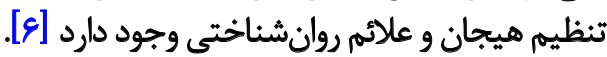
تا به امروزء تحقيقاتى كه تنظيم هيجان را در زمينه اختلال

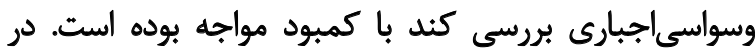

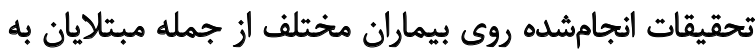

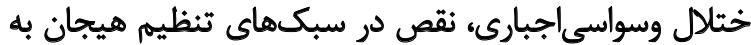

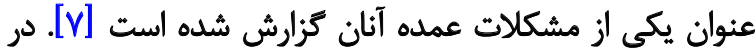

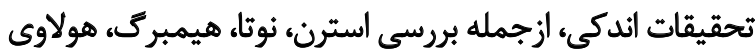

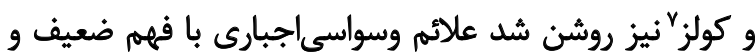

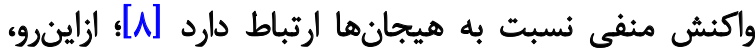

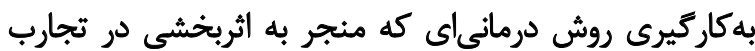

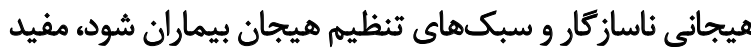

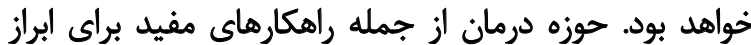
مناسبترين و بهنجارترين هيجان هوان ارائه مي ردهد.

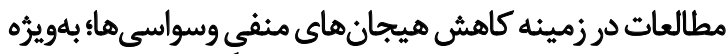

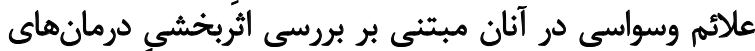

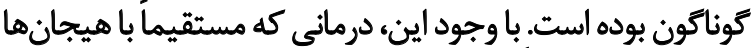

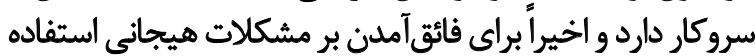

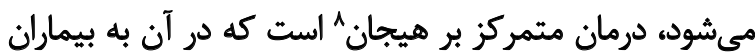

4. Panic

5. Summerfeldt, Kloosterman, Antony, Richter \& Swinson 6. Hofman \& Kashdan

7. Stern, Nota, Heimberg, Holaway \& Coles

8. Emotion-Focused Therapy (EFT) 
سوى يُروهشكَ انجام شد و مقياس ييلبراون روى آنان اجرا شد.

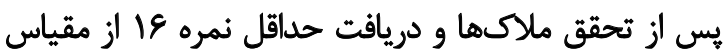

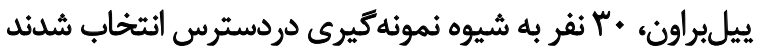

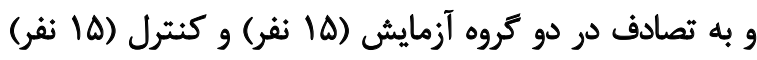

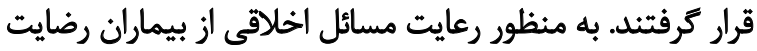

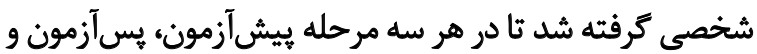

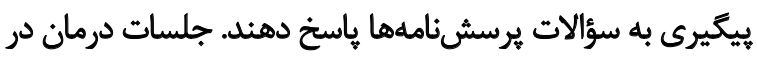

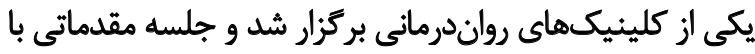

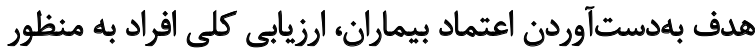

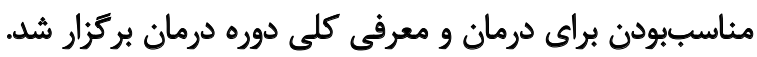

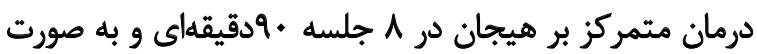

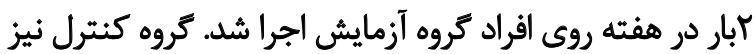

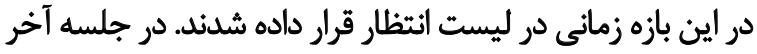
نيز از هر دو كروه يسآز آمون كرفته شد.

هيس از سيرىشدن دوره زمانى دوماهه بعد از يايان جلسات

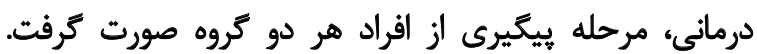

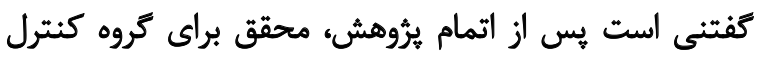

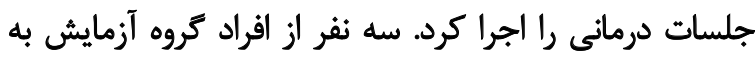

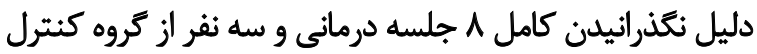

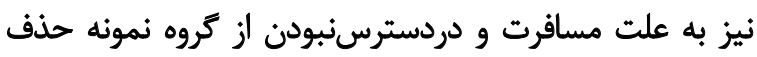

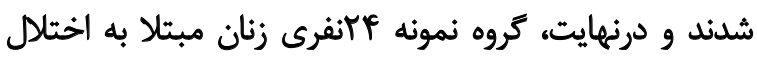

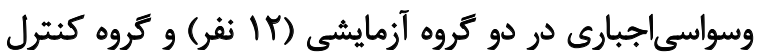

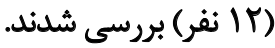

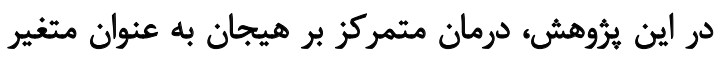

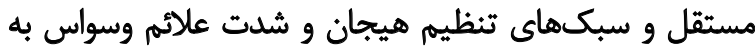

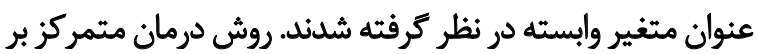

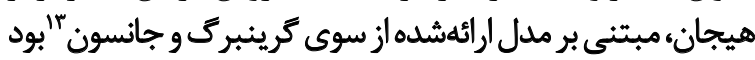

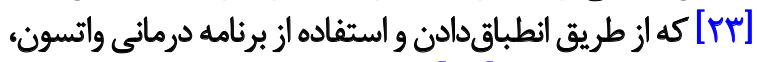

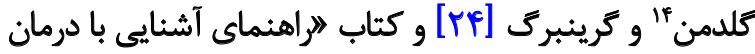

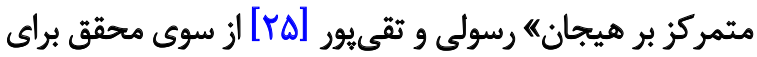

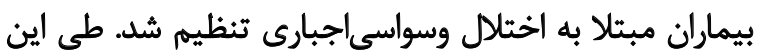

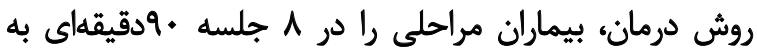

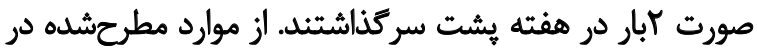

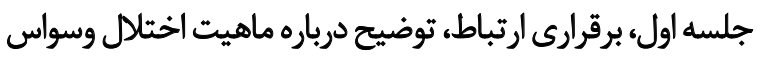
و مفهومسازى درمان متمركز بر هيجان بودئ توريان

هدف جلسات بعدى، شناسايى هيجانهاى وسواسىها،

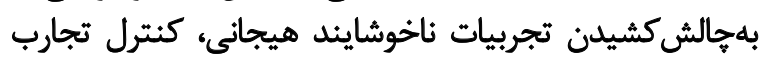

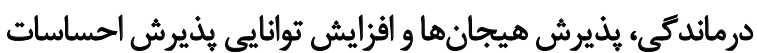

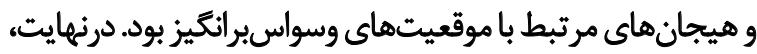

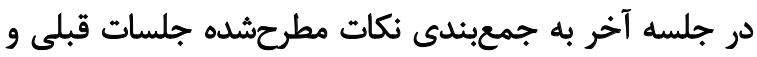

13. Johnson

14. Watson \& Goldman

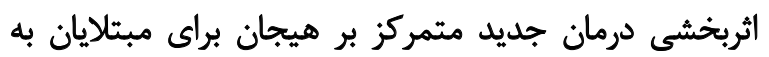

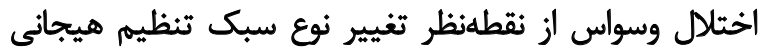

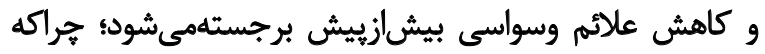

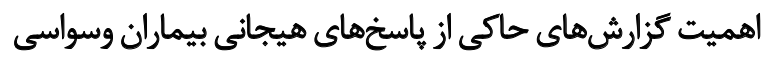

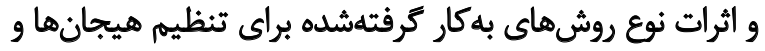

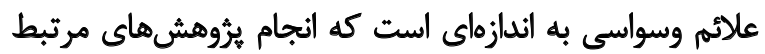

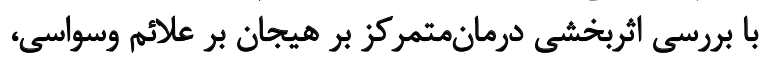

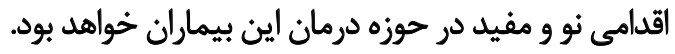
با توجه به شيوع اختلال وسواس و نقش مشكلات تنظيم

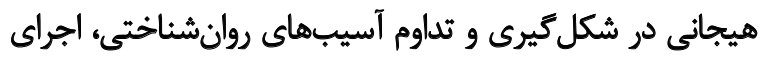

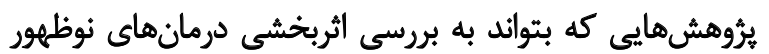

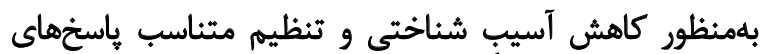

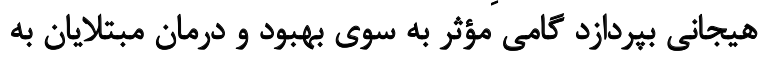

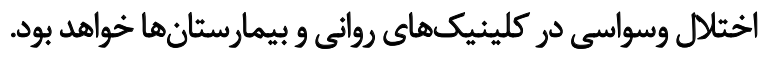

به دليل آنكه مطابق با DSM-5، زنان بزركسال بيشتر از مردان

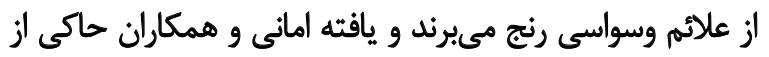

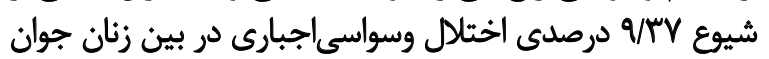

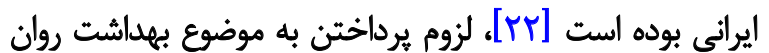

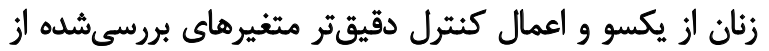

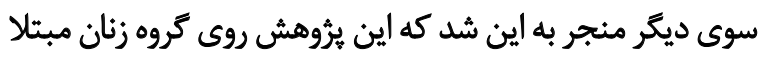

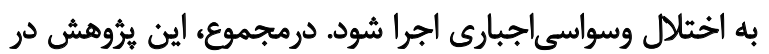

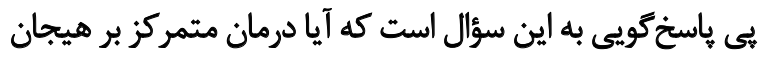

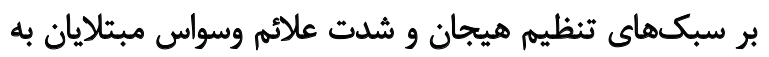

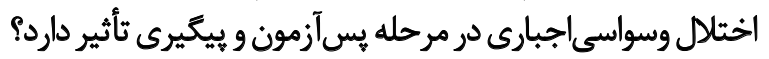

ووشُ

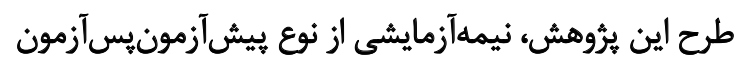

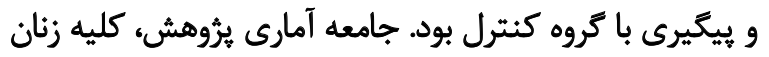

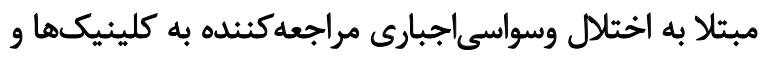

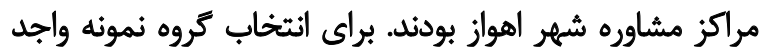

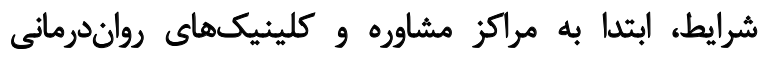

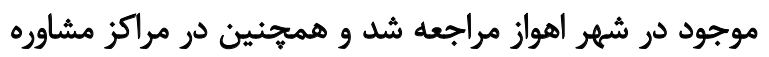

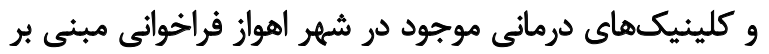

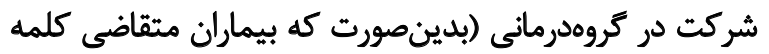

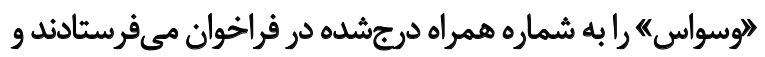

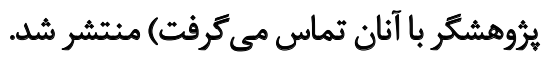

سيس براى تشخيص قطعى، با افراد واجد شرايط و و

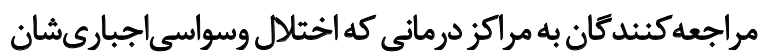

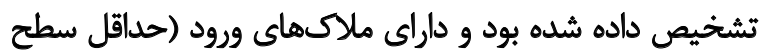

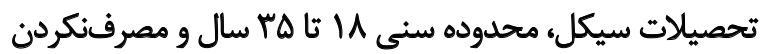

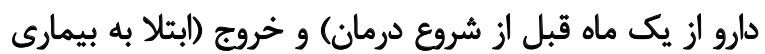

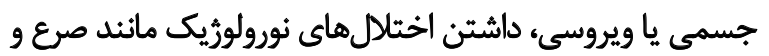

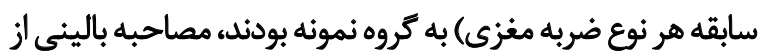




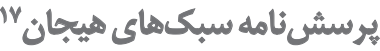

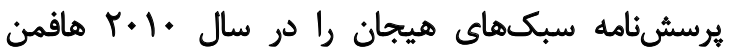

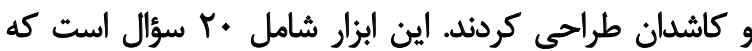

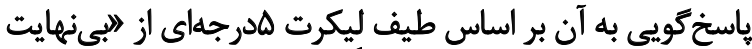

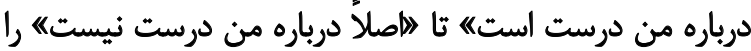

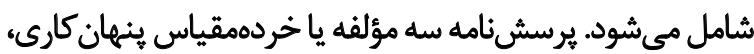

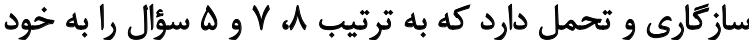

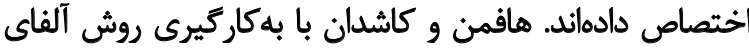

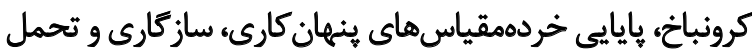

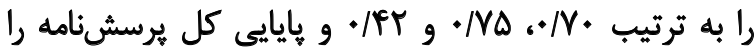

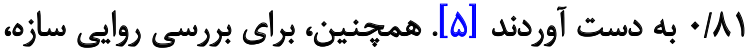

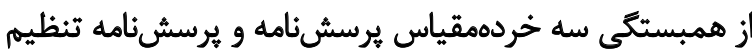

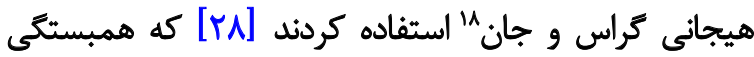

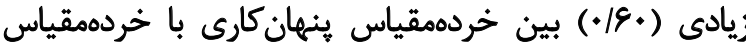

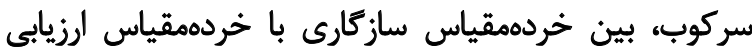

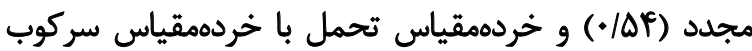

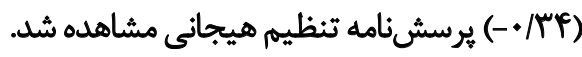

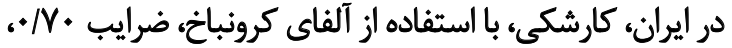

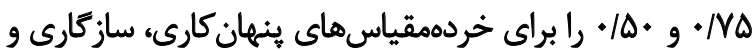

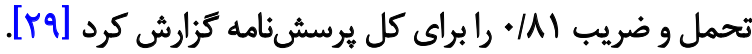

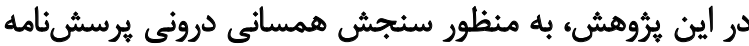

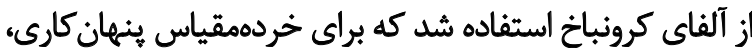
|/NF

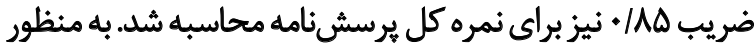

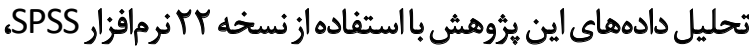

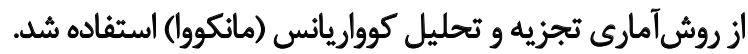

ياقتههها

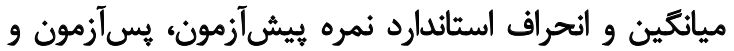

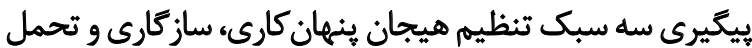

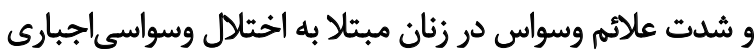

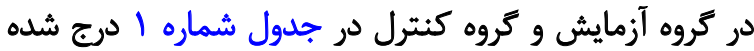

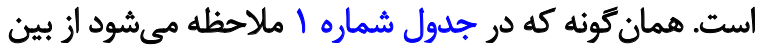
سه سبك تنظيم هيجان در كروه آزمايش، سبك دئ ينهان كاري

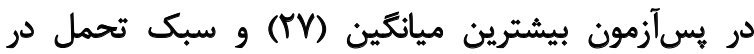

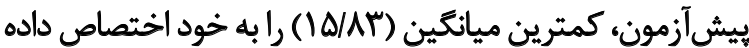

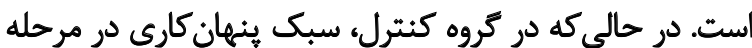

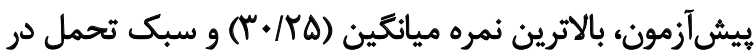

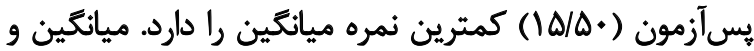

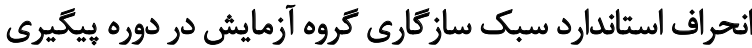

17. Affective Styles Scale (ASS)

18. Gross \& John
مرورى بر مهارتهاي ارائهشده يرداخته شد. مراحل درمان متمركز بر هيجان به صورت خلاصه در ارئه ادامه ارائه شده است. جلسه مقدماتى: بهدست آوردن اعتماد بيماران و ارزيابي كلى

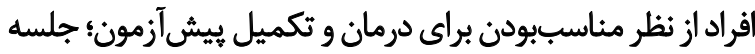

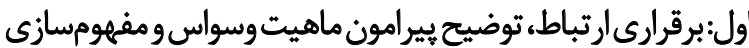

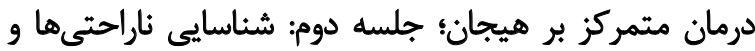

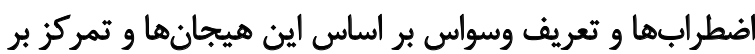

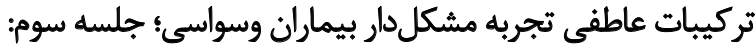

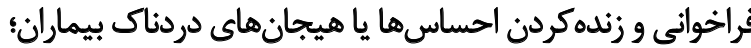

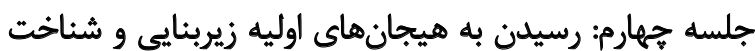

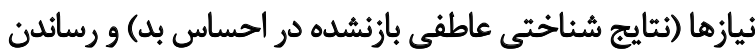

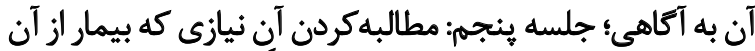

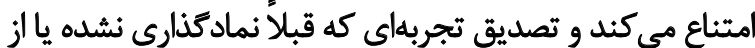

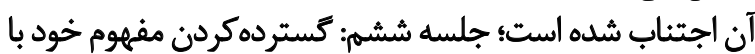

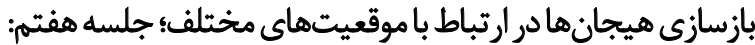

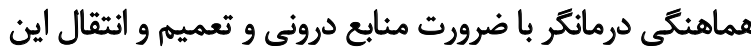

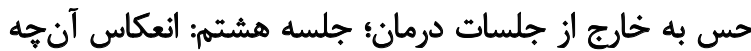

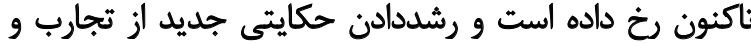

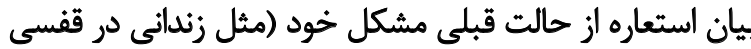

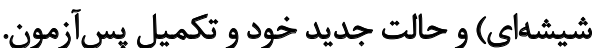

در اين يُوهش براى جمعآورى دادهها در هر سه مرحله

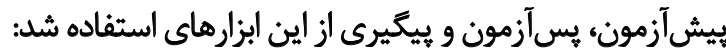

\section{مقياس وسواسى الجبارى ييل براون 19}

كودمن" و همكاران [عץ] اين فهرست را در سال 1919

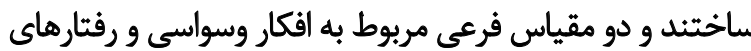

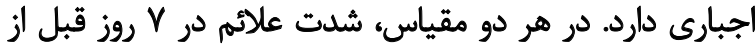

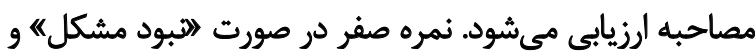

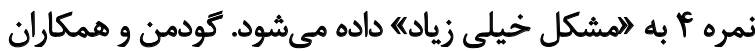

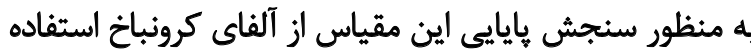

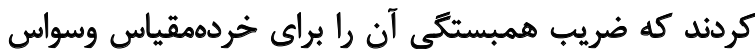

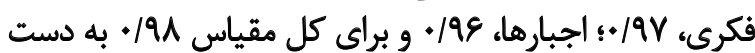

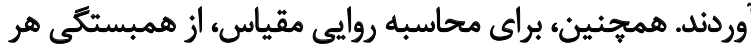

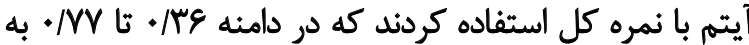

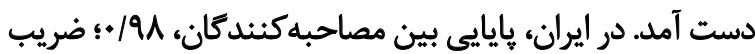

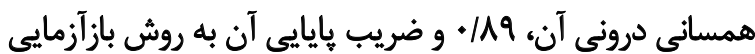

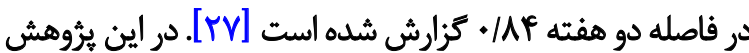

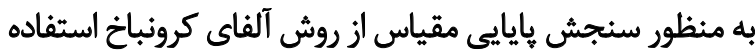

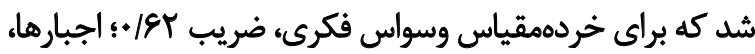

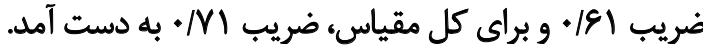

15. Yale-Brown Obsessive Compulsive Scale (Y-BOCS) 16. Goodman 
جدول ا. ميانكين و انحراف استاندارد متغيرهاى يُروهش در مراحل ييش آزمون، يس آزمون و بيكيرى

\begin{tabular}{|c|c|c|c|}
\hline \multirow{2}{*}{ ميانكين } & \multirow{2}{*}{ ميانكين تروهانحراف معيار } & \multirow{2}{*}{\multicolumn{2}{|c|}{ سبكهاى تنظيم هيجان }} \\
\hline & & & \\
\hline$r+/ r \Delta \pm r / \Delta \mid$ & $r \varphi \pm \psi / m y$ & ييش آزمون & \\
\hline$r \Delta / \Delta \Lambda \pm \Psi / T r$ & $r V \pm r / q F$ & يس آزهون & سبك ينهان كارى \\
\hline$r E \pm r / \Delta V$ & $r \vee \pm r / q)$ & ييكيرى & \\
\hline$T \Delta / \Gamma T \pm \Delta / Q T$ & $r Y / A Y \pm \Delta / 9$. & ييش آزمون & \\
\hline$r \Delta / \Delta \cdot \pm T / T r$ & $r \Delta / r \Delta \pm \Psi / \cdot r$ & يّسآزمون & سبك ساز كارى \\
\hline$r \varphi / q r \pm r / e r$ & $r \& / \cdot \wedge \pm T / P V$ & ييكيرى & \\
\hline $1 \Delta / V \Delta \pm T / A F$ & $\mid Q / A T \pm \Psi / T V$ & ييش آزمون & \\
\hline $\mid \Delta / \Delta \cdot \pm \pi / T$ & $|V / r \Psi \pm T / M|$ & يسآزمون & سبك تحمل \\
\hline $\mid \& / I Y \pm r / \varepsilon q$ & $|V| \cdot \wedge \pm r / 9 \mid$ & ينيكيرى & \\
\hline $19 / \Delta \Lambda \pm r / 4$. & $r / / Y \pm r / M$ & ييش آزمون & \\
\hline$r \cdot 1 \cdot \wedge \pm \Delta / M A$ & IF/Ta土T/AF & يس آزمون & شدت علائم وسواس \\
\hline$r \cdot / r \Psi \pm \Delta / r^{\infty} \Delta$ & $|f / T \Delta \pm T / M|$ & ييكيرى & \\
\hline
\end{tabular}

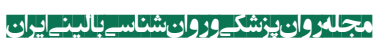

يافتههاى مربوط به آزمون لوين براى سبك ينهان اركارى،

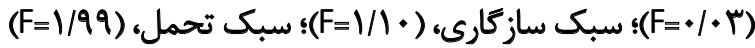

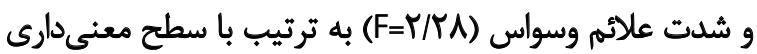
(P=./AV)

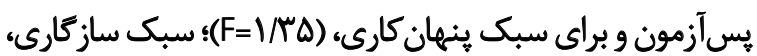
(F= I/TV)

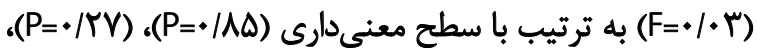

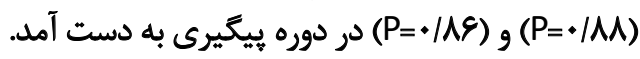

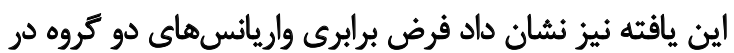

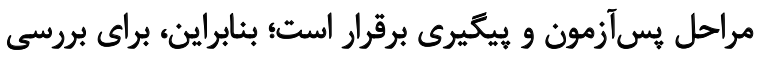

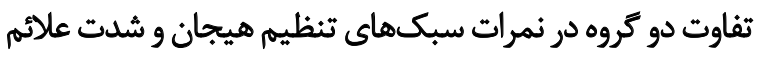

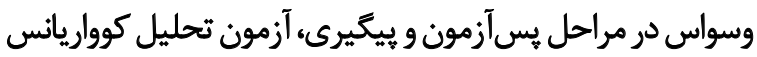

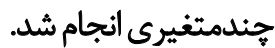

براى تعيين معنى دارى اثر كروه بر متغيرهاى سبكهان

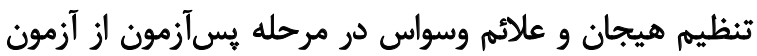

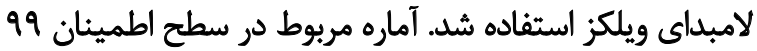

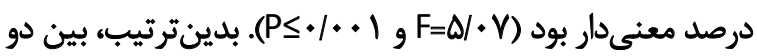

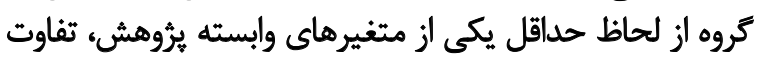

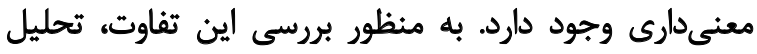

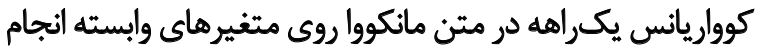

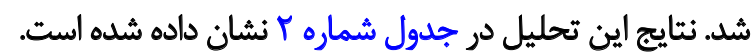

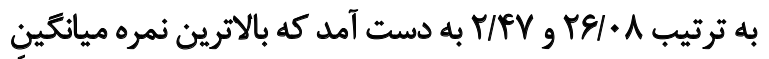

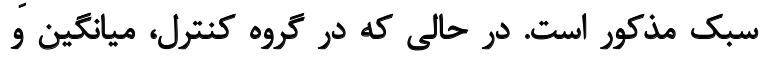

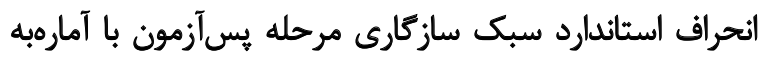

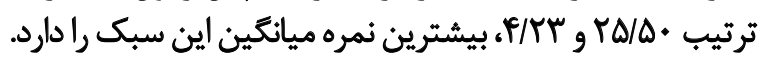

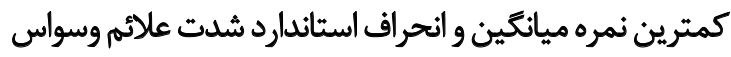

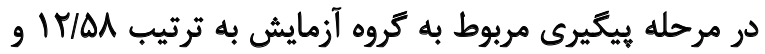

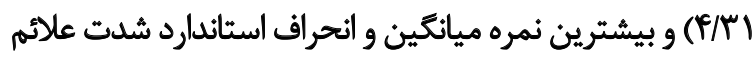

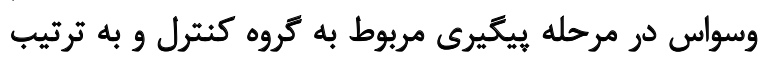

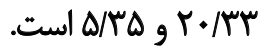

به منظور بررسى تفاوت دو كروه در نمرات سبكهاى تنظيم

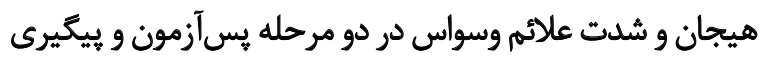

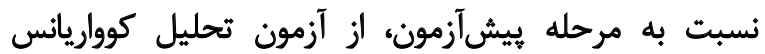

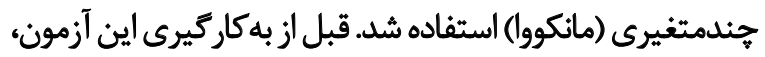

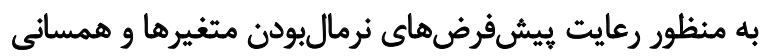

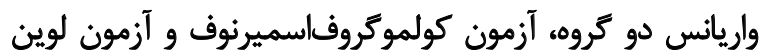

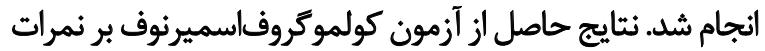

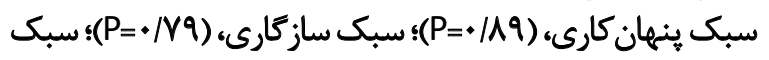

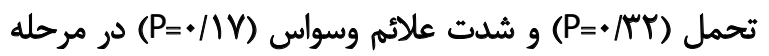

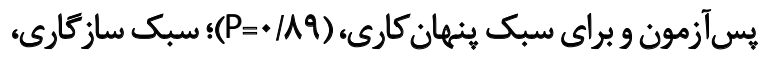
(P=॰/V9) (P=./9V) 
جدول r.نتايج تحليل كوواريانس يكراهه روى ميانكين نمرههاى يس آزمون با كنترل بيشآزمون

\begin{tabular}{|c|c|c|c|c|c|}
\hline اتدازه اثر & معنادارى & $F$ & df & متغير وابسته & 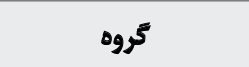 \\
\hline . NA & $\% / 1$ & $v / v$ & 1 & ينهان كارى & \\
\hline.$/ N$ &.$/ 4 r$ & .94 & 1 & ساز كارى & سبكهاى تنظيم هيجان \\
\hline.$M I$ & $\cdot / \pi r$ & $1 / 90$ & 1 & تحمل & \\
\hline.$/ 99$ & $+1+1$ & $r \cdot / F A$ & 1 & & شدت علائم وسواس \\
\hline
\end{tabular}

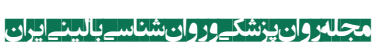

تحليل كوواريانس يكراهه در متن مانكووا روى متغيرهاى وابسته

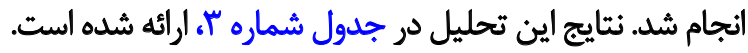

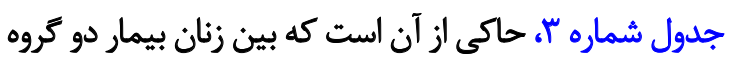

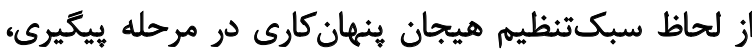

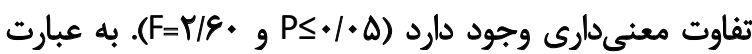

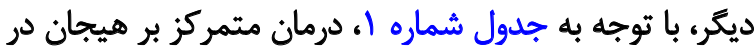

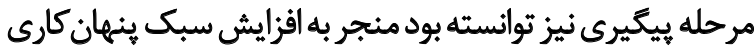

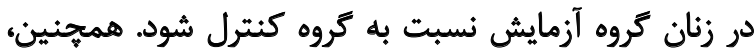

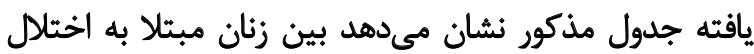

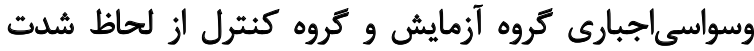

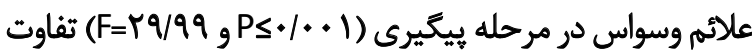

$$
\text { معنى دارى وجود دارد. }
$$

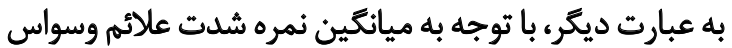

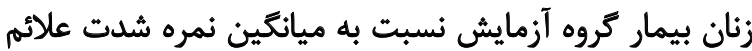

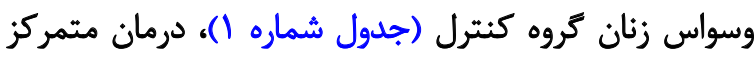

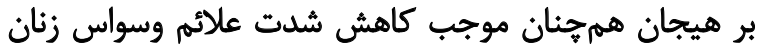

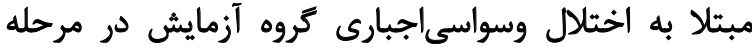

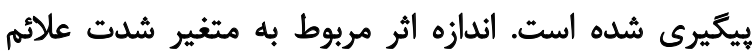

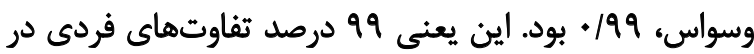

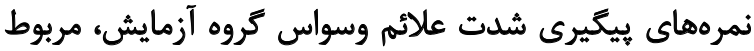

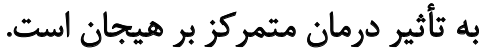

نتايج مندرج در جدول شماره Y نشان مى دهد بين زنان مبثلا

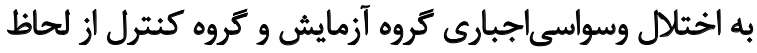

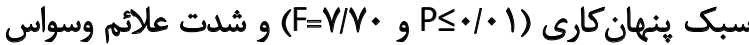

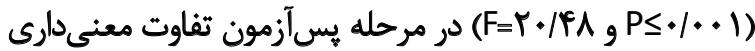

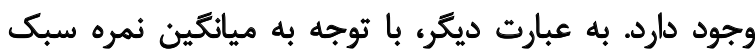

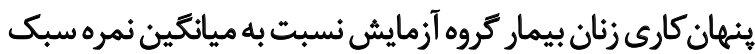

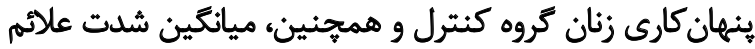

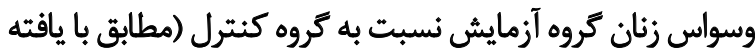

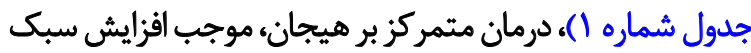

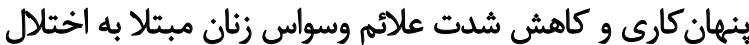

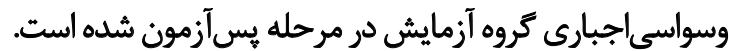
مطابق جدول شماره با، ميزان تأثير مداخله براى سبك برائ

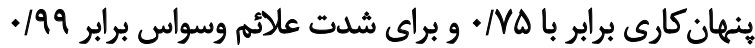

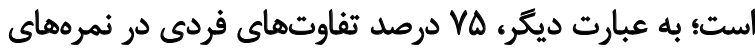

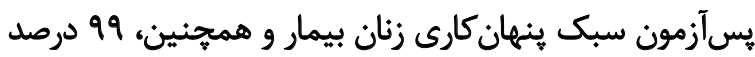

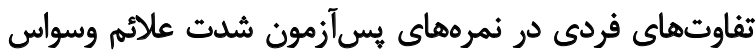

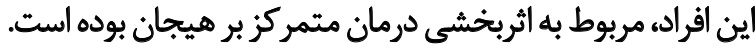
براى تعيين معنى دارى اثر كروه بر متغيرهاي سبكهاي

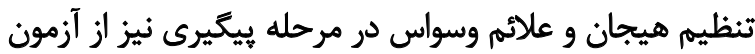

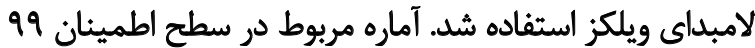

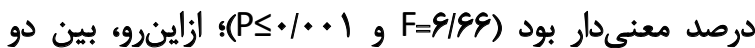

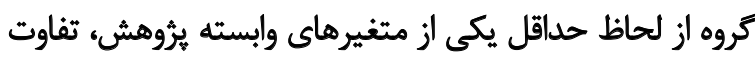

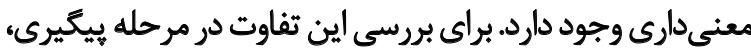

جدول ب. نتايج تحليل كوواريانس يكراهه روى ميانكين نمرههاى بيكيرى با كنترل بيش آزمون

\begin{tabular}{|c|c|c|c|c|c|}
\hline الدازه اثر & معنادارى & $F$ & df & هتغير وابسته & 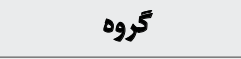 \\
\hline.$/ \pi$ & .1 .0 & $r / 8$. & 1 & ينهان كارى & \\
\hline$/ \pi$ & 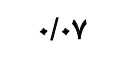 & $r / N$ & 1 & سازكارى & سبكهاى تنظيم هيجان \\
\hline . TA & .110 & $r / I$ & 1 & تحمل & \\
\hline .199 & $.1 . .1$ & หจ/१९ & 1 & & شدت علائم وسواس \\
\hline
\end{tabular}


راهحل هاى عملى مسئلهمدار در بيش كيرند. از آنجا كه ميانكين

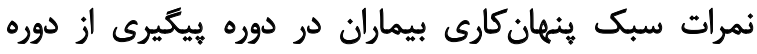

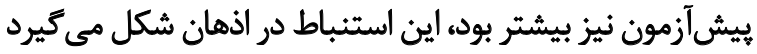

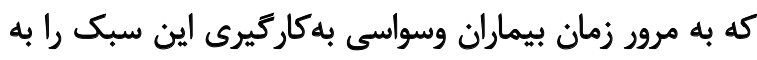

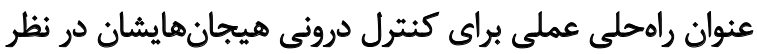

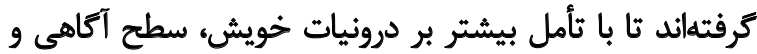
مسئوليتيذيرى خود را افزايش دهند.

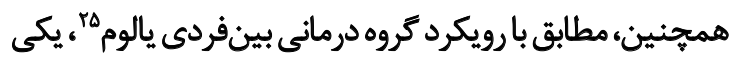

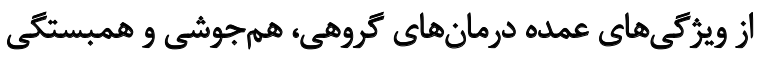

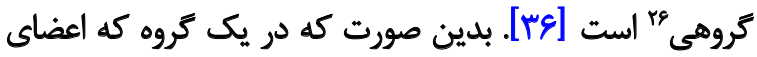

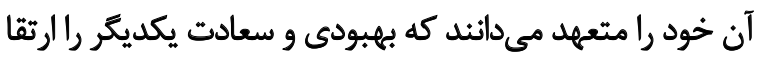

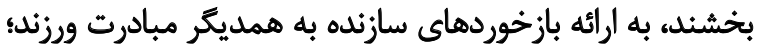

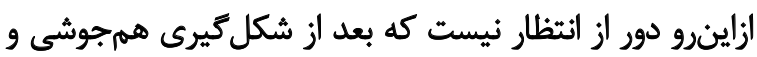

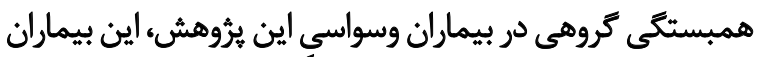

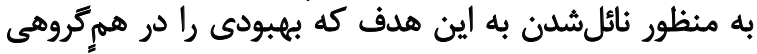

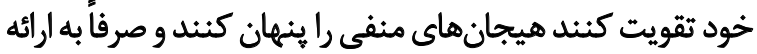

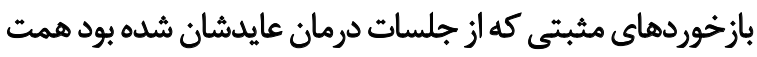

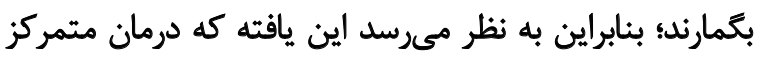

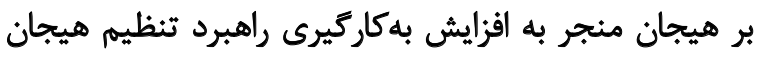

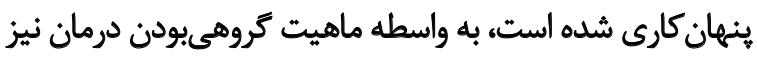
بوده است.

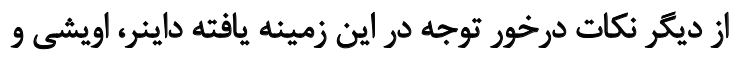

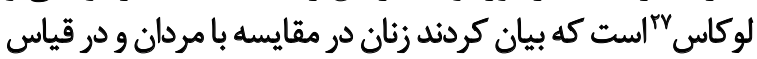

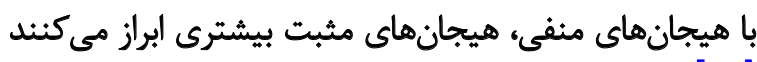

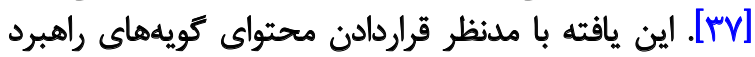

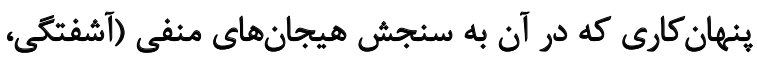

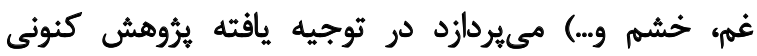

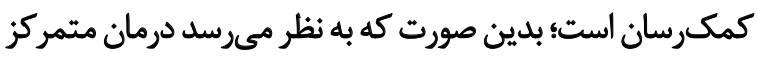

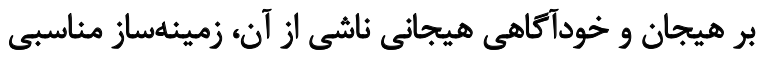

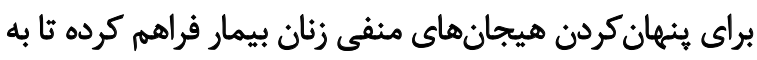

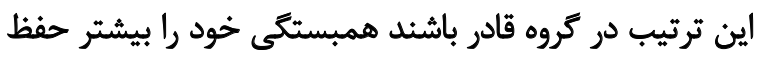
كنئد

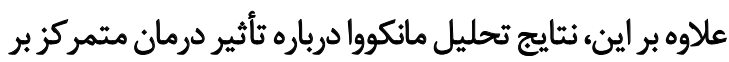

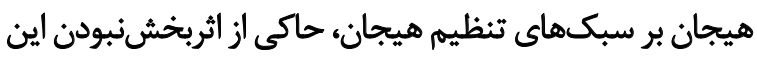

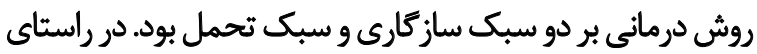

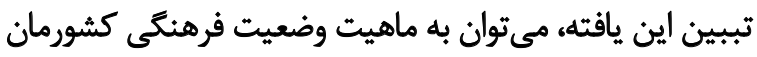

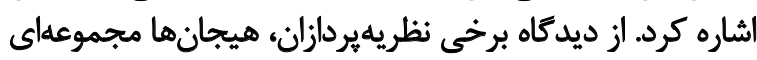

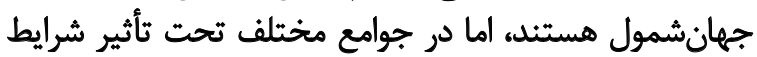

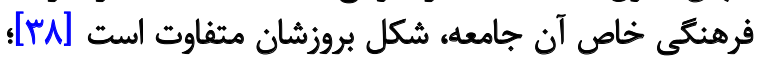

25. Yalom

26. Group cohesion

27. Diner, Oishi \& Lucac
ب

هدف اين يُروهش، بررسى اثربخشى درمان متمركز بر هيجان

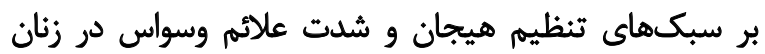

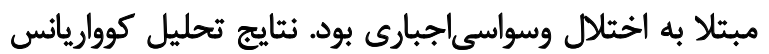

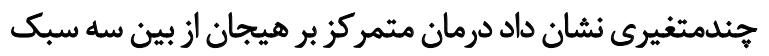

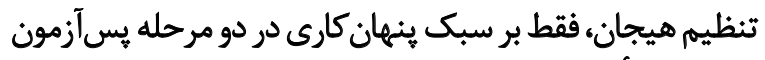

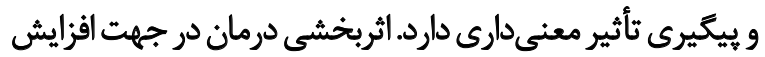

سبك ينهان كارى بود.

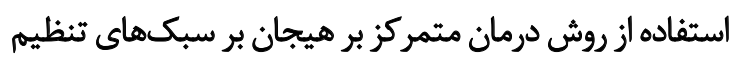

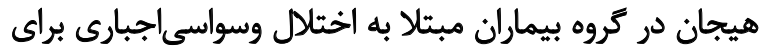

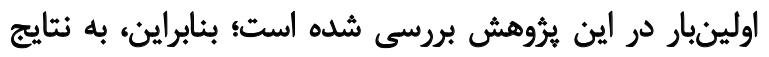

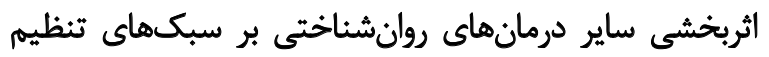

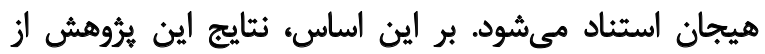

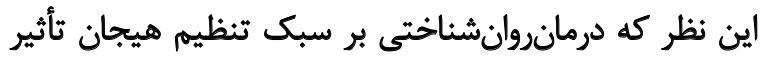

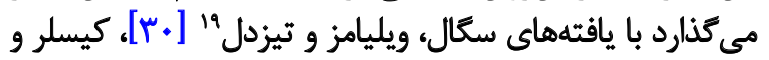

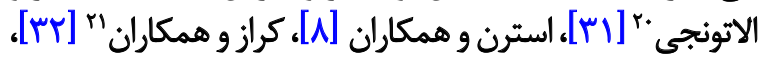

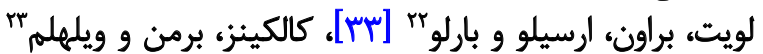

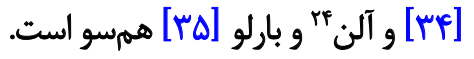

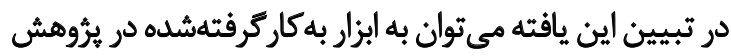

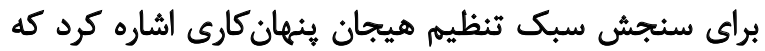

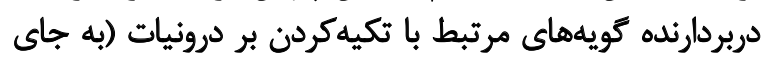

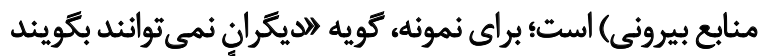

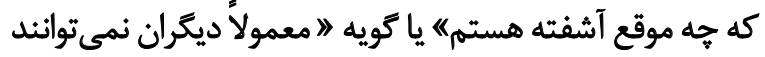

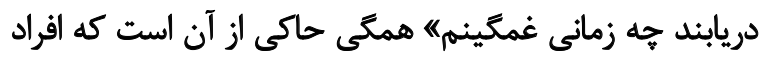

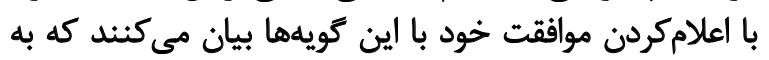

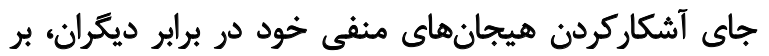

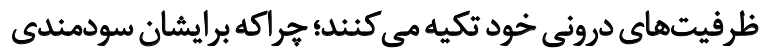

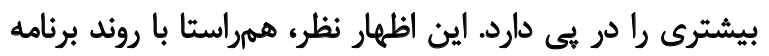

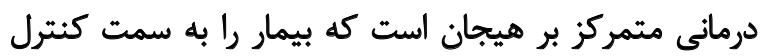

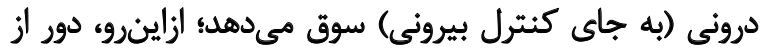

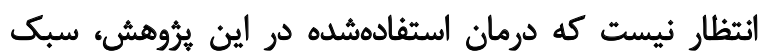
تنظيم هيجان ينهان كارى را افزايش داده باشد.

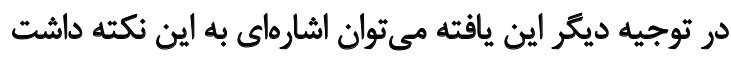

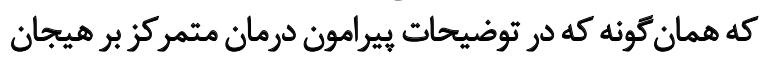

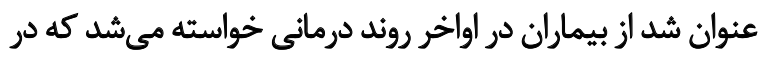

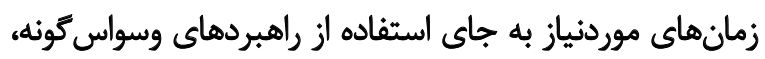

19. Segal, Williams \& Teasdale

20. Cisler \& Olatunji

21. Cruz

22. Levitt, Brown, Orsillo \& Barlow

23. Calkins, Berman \& Wilhelm

24. Allen 
اسكووارتز و فار وَاين دو احساس را به عنوان فاكتورهايي مهرم

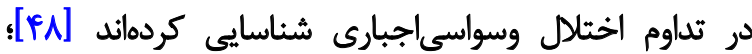

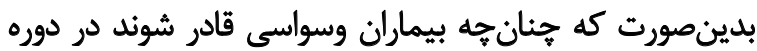

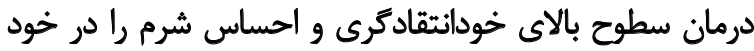

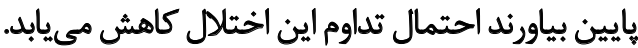

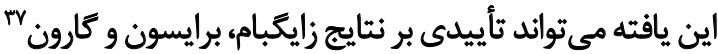

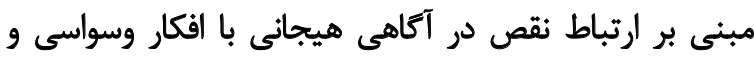

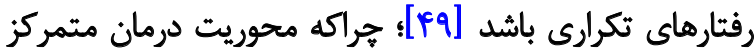

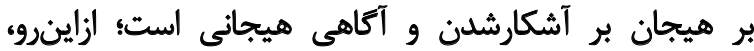

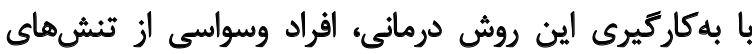

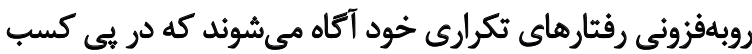

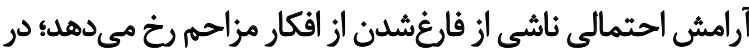

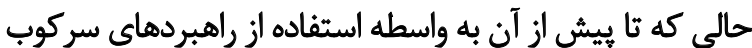

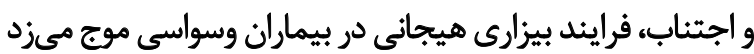

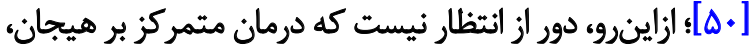

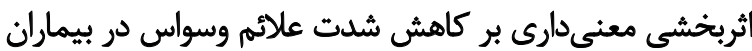
مبتلا به اختلال وسواسى اجبارى به همراه داشت داشته باشد. اتلت ^"و همكاران، اجتناب از آسيب را سازماي معرفي كردند

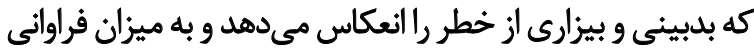

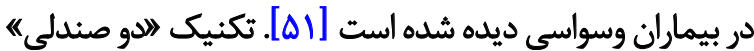

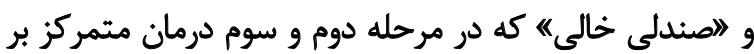

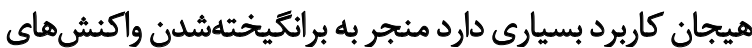

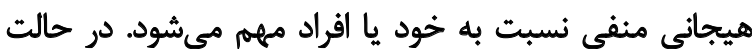

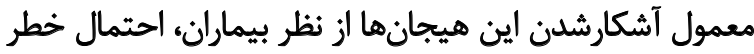

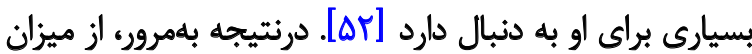

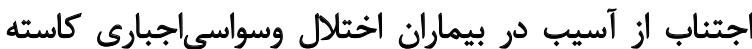

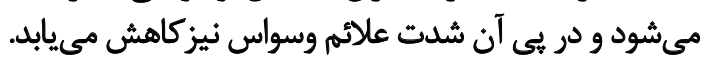
در تبيين ديكر اين يافته، شايد بتوان به اين نكته اشاره كرد

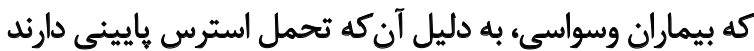

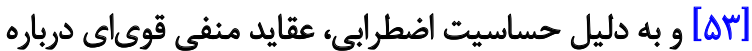

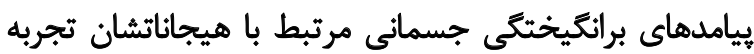

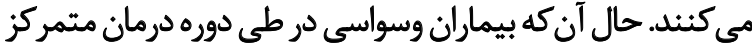

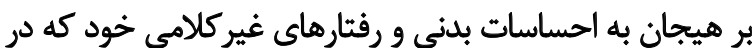

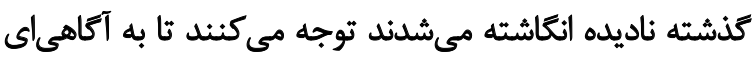

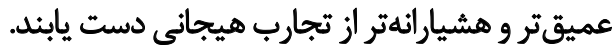
در مراحل يايانى نيز با عقايد منفى بيرامون افكار ناكارآمد

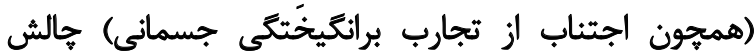

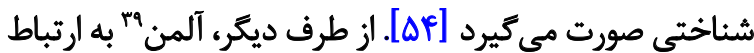

36. Abramowitz, Franklin, Schwartz \& Furr

37. Zwaigenbaum, Bryson \& Garon

38. Ettelt

39. Aleman
ازاينرو، تفاوتهاى فرهنكى در فرايندهاى تنظيم هيجانى وجود

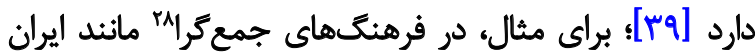

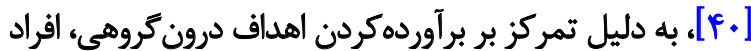

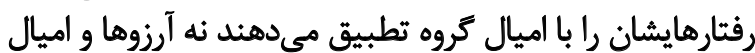

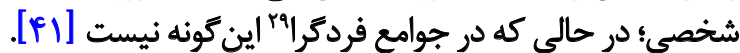
به نظر محرسد اهداف كروه درمانى ايجاب مى كرده اعضايش

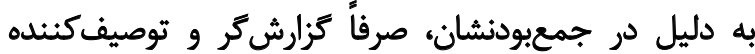

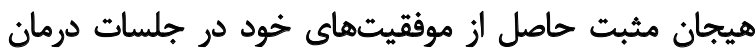

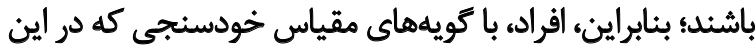

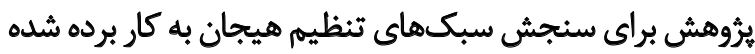

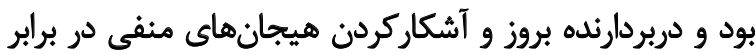

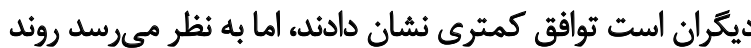

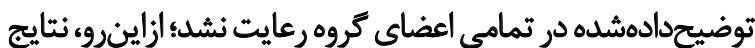

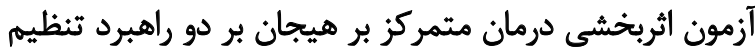

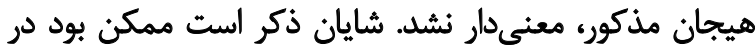

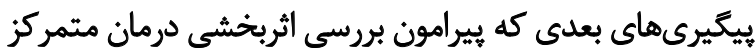

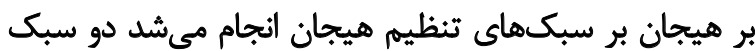

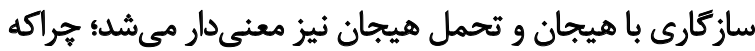

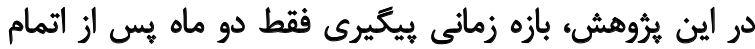

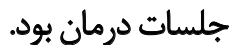

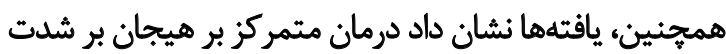

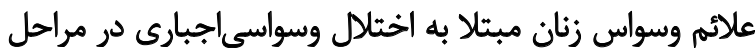

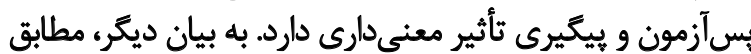

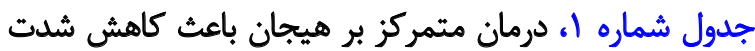

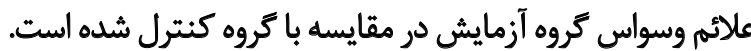

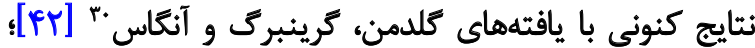

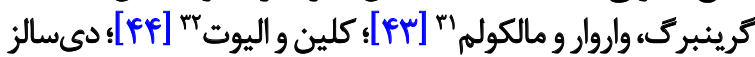

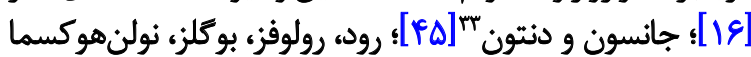

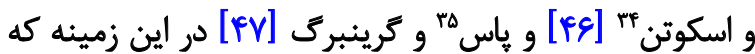

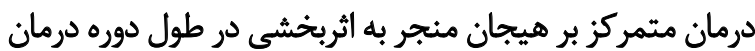

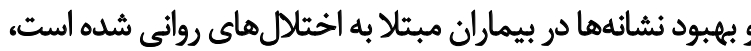

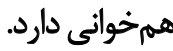

در توجيه اين يافته مي توان كفت درمان متمركز بر هيجان

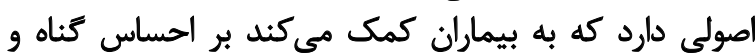

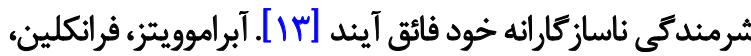

28. Collectivistic cultures

29. Individualistic cultures

30. Angus

31. Warwar \& Malcolm

32. Klein \& Elliott

33. Dessaulles, Johnson \& Denton

34. Rood, Roelofs, Bogels, Nolen-Hoeksema \& Schouten 35. Pos 


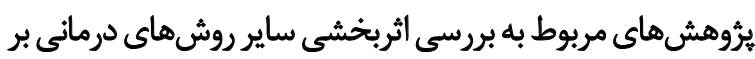

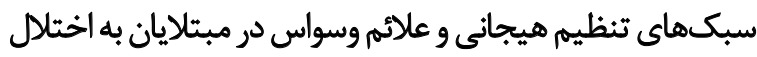

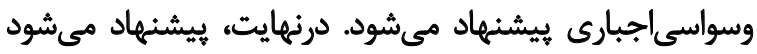

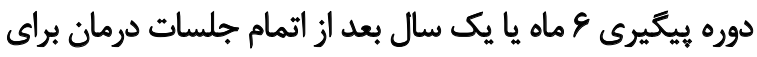
بررسى عميقتر اثربخشى درمان متمركز بر هيجان اجرا شوان بعان

ملاحظاث اخلاقي

\section{يبيروى ائ اصول الخاق يؤوهش}

در اين يُروهش بيماران فرم رعايت نكات اخلاقى را تكميل و ئروهشكران آن رارعايت كردند.

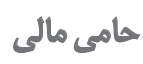

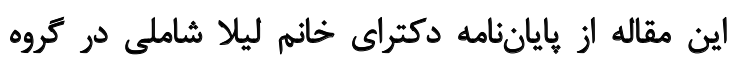

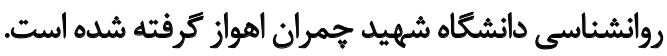

$$
\text { مشاركت ثويسند مَّان }
$$

مشاركت نويسندكان مقاله به اين شرح است: مفهومسازى:

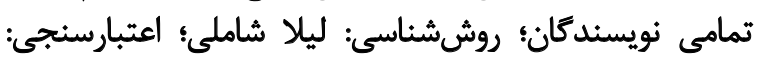

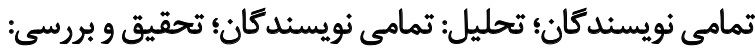

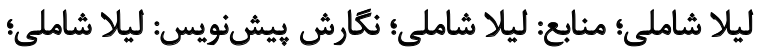

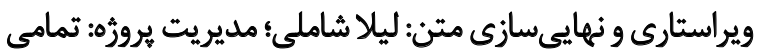

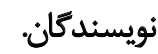

$$
\text { تعارض مثافع }
$$

بنا به اظهار نويسنده مسئول، اين مقاله تعارض منافع ندارد.

$$
\text { سباسيكّز إىى }
$$

در بايان از مدير مسئول مركز مشاوره طراوت كه با بائ

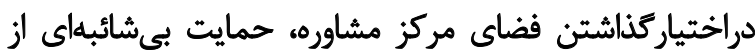
هيروهشكران داشتند تشكر مى كنيهم.
نارسايى هيجانىاى اشاره كرده است كه در توصيف و شناسايى إنائ

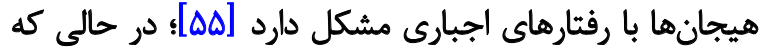

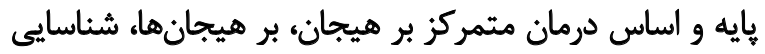

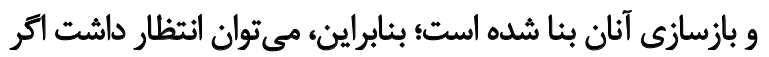

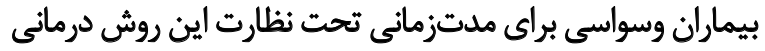
قرار بخيرند، علائم بيمارى در آنان كاهش يابد يابد.

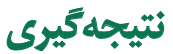

درمان متمركز بر هيجان، باعث افزايش سبك تنظيم هيجان

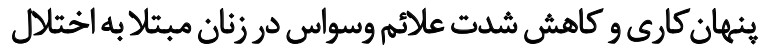

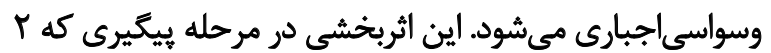

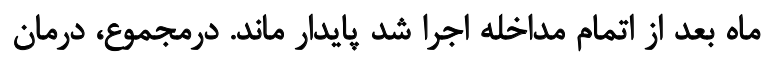

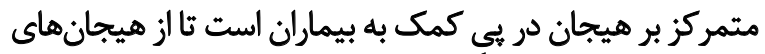

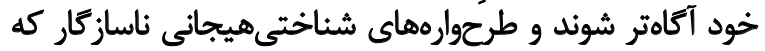

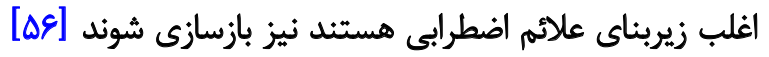

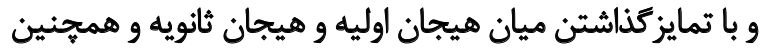

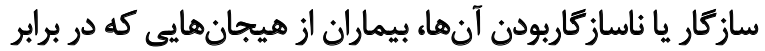

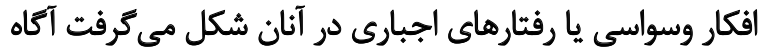

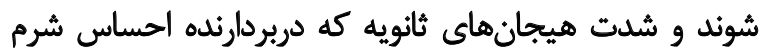
وكتياه بابت رخنه كردن غيرارادى افكار مزاحهم بود كاهش يابد.

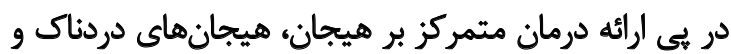

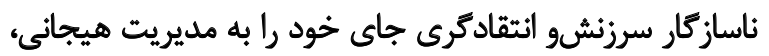

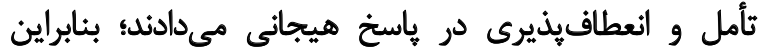

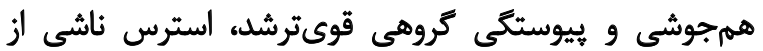

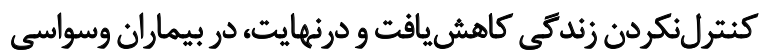

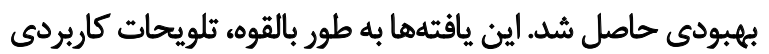

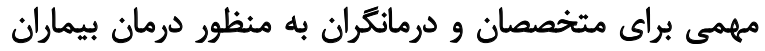

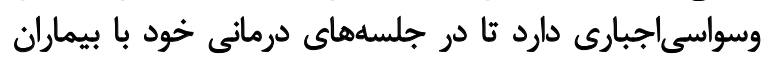

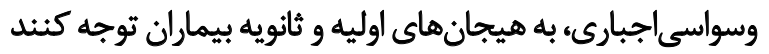

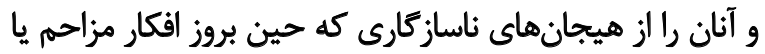

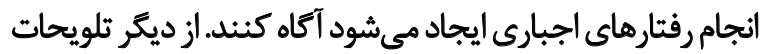

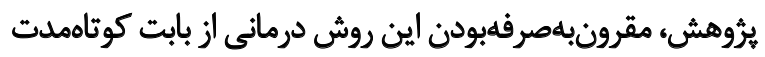

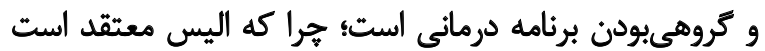

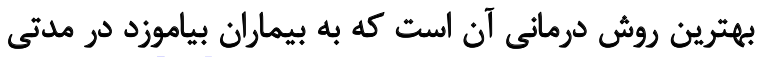

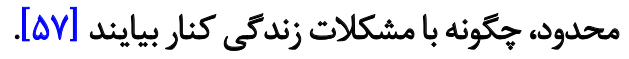

هر يزوهشى در مراحل مختلف خود با محدوديتهايى مواجه

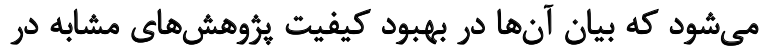

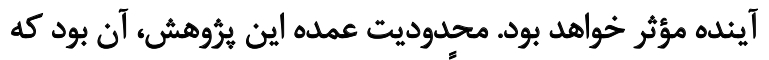

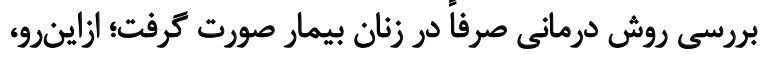

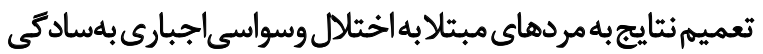

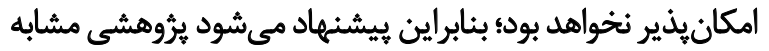

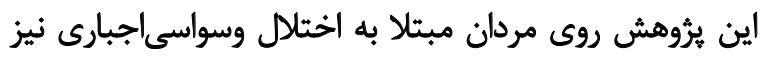

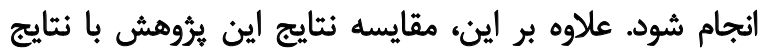




\section{References}

[1] American Psychiatric Association. Diagnostic and Statistical Manual of mental disorders (DSM-5). Washington: American Psychiatric Association; 2013

[2] Summerfeldt LJ, Kloosterman PH, Antony MM, Richter MA, Swinson RM. The relationship between miscellaneous symptoms and major symptom factors in obsessive-compulsive disorder. Behaviour Research and Therapy. 2004; 42(12):1453-67. [DOI:10.1016/j.brat.2003.09.006] [PMID]

[3] Mennin DS, Heimberg RG, Turk CL, Fresco DM. Preliminary evidence for an emotion dysregulation model of generalized anxiety disorder. Behaviour Research and Therapy. 2005; 43(10):1281310. [DOI:10.1016/j.brat.2004.08.008] [PMID]

[4] Blair KA, Denham SA, Kochanoff A, Whipple B. Playing it cool: Temperament, emotion regulation, and social behavior in preschoolers. Journal of School Psychology. 2004; 42(6):419-43. [DOI:10.1016/j.jsp.2004.10.002]

[5] Hofmann SG, Kashdan TB. The affective style questionnaire: Development and psychometric properties. Journal of Psychopathology and Behavioral Assessment. 2010; 32(2):255-63. [DOI:10.1007/s10862-009-9142-4] [PMID] [PMCID]

[6] Aldao A, Nolen Hoeksema S, Schweizer S. Emotion-regulation strategies across psychopathology: A meta-analytic review. Clinical Psychology Review. 2010; 30(2):217-37. [DOI:10.1016/j. cpr.2009.11.004] [PMID]

[7] Tong BS. Emotion regulation among individual with obsessivecompulsive disorder [PhD dissertation]. Hong Kong: The University of Hong Kong; 2011.

[8] Stern MR, Nota JA, Heimberg RG, Holaway RM, Coles ME. An initial examination of emotion regulation and obsessive compulsive symptoms. Journal of Obsessive-Compulsive and Related Disorders. 2014; 3(2):109-14. [DOI:10.1016/i.jocrd.2014.02.005]

[9] Greenberg LS. Emotion-focused therapy: Coaching clients to work through feelings. Washington: American Psychological Association; 2002. [DOI:10.1037/10447-000]

[10] Greenberg LS. Emotion-focused therapy: A clinical synthesis. Psychotherapy. 2010; 8(1):32-42. [DOI:10.1176/foc.8.1.foc32]

[11] Mennin DS, Farach F. Emotion and evolving treatments for adult psychopathology. Clinical Psychology: Science and Practice. 2007; 14(4):329-52. [DOI:10.1111/j.1468-2850.2007.00094.x]

[12] Soltani M, Shairi MR, Roshan R, Rahimi CH. The impact of emotionally focused therapy on emotional distress in infertile couples. International Journal of Fertility \& Sterility. 2014; 7(4):337-44. [PMID]

[13] Greenberg LS. Emotion-focused therapy: A synopsis. Journal of Contemporary Psychotherapy. 2006; 36(2):87-93. [DOI:10.1007/ s10879-006-9011-3]

[14] Sloan DM. Emotion-focused therapy: An interview with Leslie Greenberg. Journal of Contemporary Psychotherapy. 2004; 34(2):105-16. [DOI:10.1023/B:JOCP.0000022310.34573.b7]

[15] MacKinnon RA, Michels R, Buckley PJ. The psychiatric interview in clinical practice. Washington: American Psychiatric Association; 2016.
[16] Elliott R. Emotion-focused therapy. In Sanders P, editors. The tribes of the person-centred nation: An introduction to the schools of therapy related to the person-centred approach. Wyastone: PCCS Books; 2012.

[17] Carryer JR, Greenberg LS. Optimal levels of emotional arousal in experiential therapy of depression. Journal of Consulting and Clinical Psychology. 2010; 78(2):190-9. [DOI:10.1037/a0018401] [PMID]

[18] Ellison JA, Greenberg LS, Goldman RN, Angus L. Maintenance of gains following experiential therapies for depression Journal of Consulting and Clinical Psychology. 2009; 77(1):103-12. [DOI:10.1037/a0014653] [PMID]

[19] Shahar B. Emotion-focused therapy for treatment of social anxiety: An overview of the model and a case description. Clinical Psychology \& Psychotherapy. 2014; 21(6):536-47. [PMID]

[20] Pos AE, Greenberg LS., Warwar Sh. Testing a model of change in the experiential treatment of depression. Journal of Consulting and Clinical Psychology. 2009; 77(6):1055-66. [DOI:10.1037/ a0017059] [PMID]

[21] Eddy KT, Dutra L, Bradley R, Westen D. A multidimensiona meta-analysis of psychotherapy and pharmacotherapy for obsessive-compulsive disorder. Clinical Psychology Review. 2004; 4(8):1011-30. [DOI:10.1016/j.cpr.2004.08.004] [PMID]

[22] Amani M, Abolghasemi A, Ahadi B, Narimani M. [The prevalence of obsessive-compulsive disorder among the women 20 to 40 years old of Ardabil city, Western part of Iran (Persian)]. Journal of Fundamentals of Mental Health. 2013; 15(3):32-41.

[23] Greenberg LS, Johnson S. Emotionally focused couples' therapy. New York: Guilford Press; 1988.

[24] Watson SS, Goldman RN, Greenberg LS. Contrasting two clients in emotion-focused therapy for depression. Pragmatic Case Studies in Psychotherapy. 2011; 2(3):266-364.

[25] Rasooli R, Taghipour E. [Familiarize with Emotion-focused Therapy (Persian)]. Tehran: Roshd-e Farhang; 2010.

[26] Goodman WK, Price LH, Rassmussen SA, Mazure C, Delgado P, Heninger GR, et al. Yale-brown obsessive-compulsive scale: Development, use and reliability. Archives of General Psychiatry. 1989; 46(11):1006-11. [DOI:10.1001/ archpsyc.1989.01810110048007] [PMID]

[27] Dadfar M, Bolhari J, Malakooti K, Bayanzade SA. [Prevalence of the obsessive-compulsive disorder symptoms (Persian)]. Iranian Journal of Psychiatry and Clinical Psychology. 2001; 7(1):27-33.

[28] Gross JJ, John OP. Individual differences in two emotion regulation processes: Implications for affect, relationships, and well-being. Journal of Personality and Social Psychology. 2003; 85(2):348-62. [DOI:10.1037/0022-3514.85.2.348]

[29] Kareshki H. [Evaluating factorial structure of emotional styles in students (Persian)]. Journal of Research in Behavioural Sciences. 2013; 11(3):185-95.

[30] Segal ZV, Williams JMG, Teasdale JD. Mindfulness-based cognitive therapy for depression: A new approach to preventing relapse. New York: Guilford; 2002

[31] Cisler JM, Olatunji BO. Emotion regulation and anxiety disorders. Current Psychiatry Reports. 2012; 14(3):182-7. [DOI:10.1007/ s11920-012-0262-2] [PMID] [PMCID] 
[32] Cruz LF, Landau D, Iervolino AC, Santo S, Pertusa A, Singh S, et al. Experiential avoidance and emotion regulation difficulties in hoarding disorder. Journal of Anxiety Disorders. 2013; 27(2):2049. [DOI:10.1016/j.janxdis.2013.01.004] [PMID]

[33] Levitt JT, Brown TA, Orsillo SM, Barlow DH. Acceptance versus suppression of emotion on subjective and psychophysiological response to carbon dioxide challenge in patients with panic disorder. Behavior Therapy. 2004; 35(4):747-66. [DOI:10.1016/ S0005-7894(04)80018-2]

[34] Calkins AW, Berman NC, Wilhelm S. Recent advances in research on cognition and emotion in OCD: A review. Current Psychiatry Reports. 2013; 15(5):1-7. [DOI:10.1007/s11920-0130357-4] [PMID]

[35] Allen LB, Barlow DH. Relationship of exposure to clinically irrelevantemotion cues and obsessive-compulsive symptoms. Behavior Modification. 2009; 33(6):743-62. [DOI:10.1177/0145445509344180] [PMID]

[36] Yalom I. The theory and practice of group psychotherapy. New York: Basic Books; 1995.

[37] Diner E, Oishi S, Lucac RE. Personality, culture, and subjective well-being: Emotional and cognitive evaluations of life. Annual Review of Psychology. 2003; 54(1):403-25. [DOI:10.1146/annurev. psych.54.101601.145056] [PMID]

[38] Goleman D, Boyatzis R, Mckee A. Primal leadership: Realizing the power of emotional intelligence. Boston: The Harvard Business Review; 2002. [PMCID]

[39] Matsumoto D. Culture and cultural worldviews: Do verbal descriptions of culture reflect anything other than verbal descriptions of culture? Culture \& Psychology. 2005; 12(1):33-62. [DOI:10.1177/1354067X06061592]

[40] Alborzi M. [Explaining the relationship between motivation and creativity with regard to cultural differences (Persian)]. Journal of Iran Cultural Research. 2014; 7(1):29-49.

[41] Hofstede GH. Culture's consequences: Comparing values, behaviors, institutions and organizations across nations. Thousand Oaks, California: Sage Publications; 2001.

[42] Goldman RN, Greenberg LS, Angus L. The effects of adding emotion-focused interventions to the client-centered relationship conditions in the treatment of depression. Psychotherapy Research. 2006; 16(5):537-49. [DOI:10.1080/10503300600589456]

[43] Greenberg LS, Warwar SH, Malcolm WM. Differential effects of emotion-focused therapy and psycho education in facilitating forgiveness and letting go of emotional injuries. Journal of Counseling Psychology. 2008; 55(2):185-96. [DOI:10.1037/00220167.55.2.185]

[44] Klein MJ, Elliott R. Client accounts of personal change in process experiential psychotherapy: A methodologically pluralistic approach. Psychotherapy Research. 2006; 16(1):91-105. [DOI:10.1080/10503300500090993]

[45] Dessaulles A, Johnson SM, Denton WH. Emotion-focused therapy for couples in the treatment of depression: A pilot study. The American Journal of Family Therapy. 2003; 31(5):345-53. [DOI:10.1080/01926180390232266]

[46] Rood L, Roelofs J, Bogels SM, Nolen Hoeksema S, Schouten $\mathrm{E}$. The influence of emotion-focused rumination and distraction on depressive symptoms in non-clinical youth: A meta- analytic review. Clinical Psychology Review. 2009; 29(7):607-16. [DOI:10.1016/j.cpr.2009.07.001] [PMID]

[47] Pos AE, Greenberg LS. Emotion-focused therapy: The transforming power of affect. Journal of Contemporary Psychotherapy. 2007; 37(1):25-36. [DOI:10.1007/s10879-006-9031-z]

[48] Abramowitz JS, Franklin ME, Schwartz SA, Furr JM. Symptom presentation and outcome of cognitive-behavioral therapy for obsessive-compulsive disorder. Journal of Consulting and Clinical Psychology. 2003; 71(6):1049-57. [DOI:10.1037/0022006X.71.6.1049] [PMID]

[49] Zwaigenbaum L, Bryson S, Garon N. Early identification of autism spectrum disorders. Behavioural Brain Research. 2013; 25(1):133-46. [DOI:10.1016/j.bbr.2013.04.004] [PMID]

[50] Daughters SB, Lejuez CW, Kahler CW, Strong DR, Brown RA. Psychological distress tolerance and duration ofmost recent abstinence attempt among residential treatment-seeking substance abusers. Psychology of Addictive Behaviors. 2005; 19(2):208-11. [DOI:10.1037/0893-164X.19.2.208] [PMID]

[51] Ettelt S, Grabe HJ, Ruhrmann S, Buhtz F, Hochrein A, Kraft $S$, et al. Harm avoidance in subjects with obsessive-compulsive disorder and their families. Journal of Affective Disorders. 2008; 107(1-3):256-69. [DOI:10.1016/j.jad.2007.08.017] [PMID]

[52] Watson SS, Goldman RN, Greenberg LS. Contrasting two clients in emotion-focused therapy for depression. Pragmatic Case Studies in Psychotherapy. 2011; 2(3):266-364.

[53] Robinson LJ, Freeston MH. Emotion and internal experience in obsessive compulsive disorder: Reviewing the role of alexithymia, anxiety sensitivity and distress tolerance. Clinical Psychology Review. 2014; 34(3):256-71. [DOI:10.1016/j.cpr.2014.03.003] [PMID]

[54] Eells TD. Handbook of psychotherapy case formulation. New York: Guilford Press; 2007.

[55] Aleman A. Feelings you can't imagine: Towards a cognitive neuroscience of alexithymia. Trends in Cognitive Sciences. 2005; 9(12):553-5. [DOI:10.1016/j.tics.2005.10.002] [PMID]

[56] Greenberg LS. Emotion-focused therapy. Washington: American Psychological Association; 2011.

[57] Ellis A. Psychotherapy and the value of a human being. New York: Institute for Rational-Emotive Therapy; 1972. 